\title{
On optimization of manufacturing of a step-down DC-DC converter to increase integration density of elements
}

\author{
E. L. Pankratov \\ Department of Mathematical and Natural Sciences, Nizhny Novgorod State University, Nizhny Novgorod, Russia
}

\begin{tabular}{l} 
Article Info \\
\hline Article history: \\
Received Jul 7, 2020 \\
Revised Nov 10, 2020 \\
Accepted Jan 23, 2021 \\
\hline
\end{tabular}

\section{Keywords:}

Increasing of element integration rate Optimization of manufacturing Switched-capacitor step-down DC-DC

\begin{abstract}
We consider the possibility to increase the field-effect transistor's density in a switched-capacitor step-down DC-DC converter. Based on this approach we analyzed the manufacturing of the converter in a heterostructure with a special structure. Some specific sections of the heterostructure must be doped by ion implantation or by diffusion. After this procedure optimized annealing has been done. We also obtained conditions for decreasing mismatchinduced stress value in this heterostructure. An analytical approach for the analysis of heat and mass transport in multilayer structures has been introduced. The approach gives a possibility without crosslinking of solutions on interfaces between layers, taking into account (i) spatial variation of parameters of considered processes; (ii) temporal variation of parameters of considered processes; (iii) nonlinearity of considered processes; and (iv) mismatch-induced stress.
\end{abstract}

This is an open access article under the CC BY-SA license.

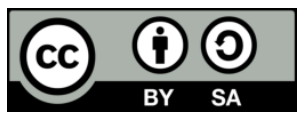

Corresponding Author:

E. L. Pankratov

Department of Mathematical and Natural Sciences

Nizhny Novgorod State University

23 Gagarin Avenue, Nizhny Novgorod, 603950, Russia

Email: elp2004@mail.ru

\section{INTRODUCTION}

Currently density of elements of integrated circuits and their performance intensively increasing. Simultaneously with increasing of the density of the elements of integrated circuit their dimensions decrease. One way to decrease dimensions of these elements of these integrated circuit is manufacturing of these elements in thin-film heterostructures [1]-[8]. An alternative approach to decrease dimensions of the elements of integrated circuits is using laser and microwave types annealing of dopant and/or radiation defects (these defects were generated during implantation of ions) [9]-[18]. Both types of annealing (laser and microwave) give a possibility to obtain inhomogeneous distribution of temperature. Inhomogeneity of temperature leads to inhomogeneity of all temperature- dependent parameters (diffusion coefficient and other) due to the Arrhenius law. The inhomogeneity of properties of materials during doping gives a possibility to decrease dimensions of elements of integrated circuits [19]. Changing of properties of electronic materials could be obtain by using radiation processing of these materials [8], [9].

In this paper we consider a cascaded-inverter circuit based on field-effect transistors described in [4] see Figure 1. We assume, that the considered element has been manufactured in heterostructure from Figure 1. The heterostructure consist of a substrate and an epitaxial layer. The epitaxial layer includes into itself several sections manufactured by using another material. The sections should be doped for generation into these sections required type of conductivity ( $n$ or $p$ ). We consider two types of doping: diffusion of dopant and implantation of ions of dopants. Framework this paper we analyzed redistribution of dopant 
during annealing of dopant and/or radiation defects to formulate conditions for decreasing of dimensions of the considered a cascaded-inverter circuit.

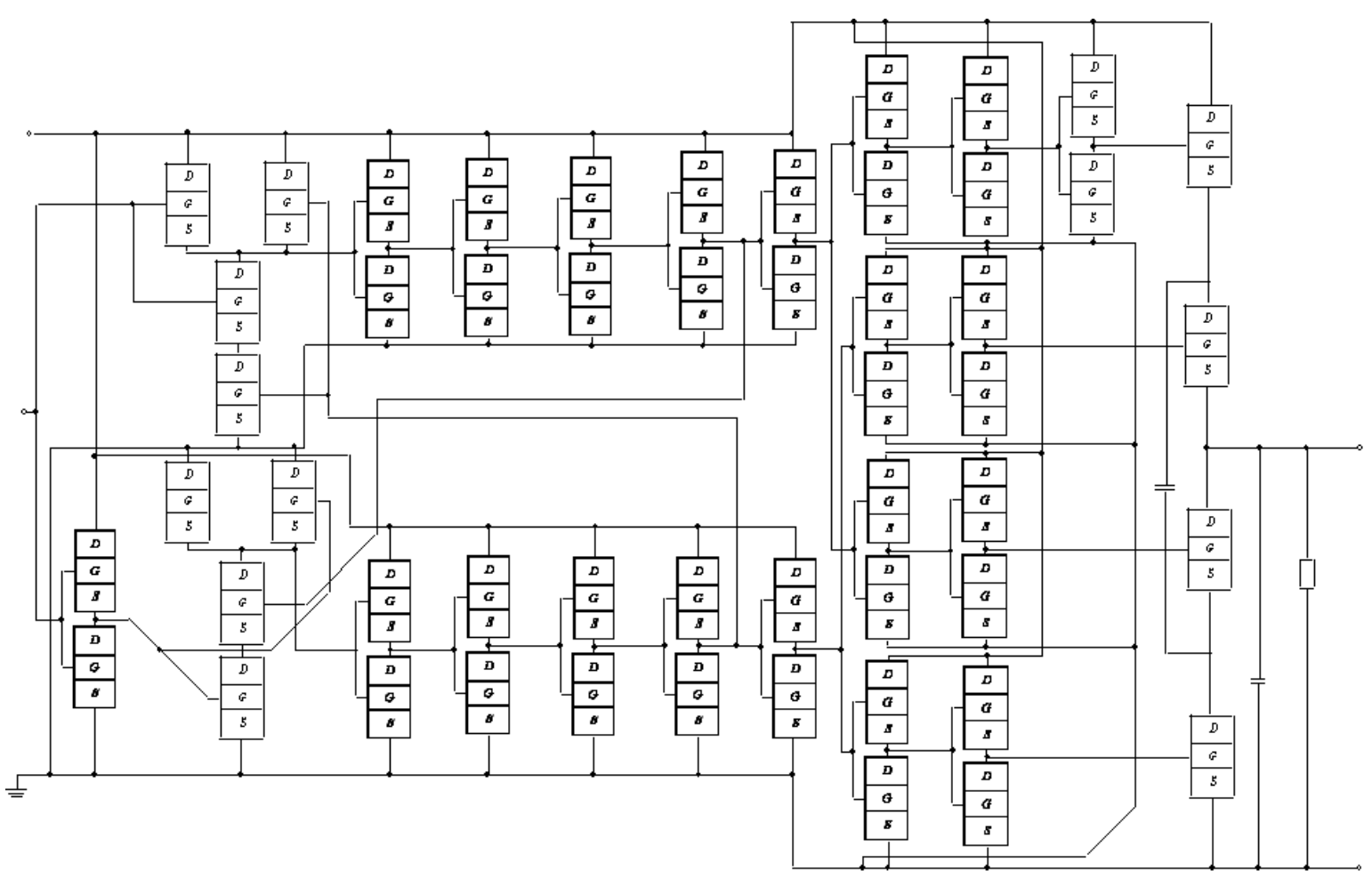

Figure 1. The considered cascaded-inverter [4]

\section{METHOD OF SOLUTION}

To solve our aim, we shall analyze spatio-temporal distribution of concentration of dopant. The distribution has been determined by solving the following boundary problem [1], [3], [19].

$$
\frac{\partial C(x, y, z, t)}{\partial t}=\frac{\partial}{\partial x}\left[D_{C} \frac{\partial C(x, y, z, t)}{\partial x}\right]+\frac{\partial}{\partial y}\left[D_{C} \frac{\partial C(x, y, z, t)}{\partial y}\right]+\frac{\partial}{\partial z}\left[D_{C} \frac{\partial C(x, y, z, t)}{\partial z}\right]
$$

Boundary and initial conditions could be written as (2).

$$
\begin{aligned}
& \left.\frac{\partial C(x, y, z, t)}{\partial x}\right|_{x=0}=\left.0 \frac{\partial C(x, y, z, t)}{\partial x}\right|_{x=L_{x}}=\left.0 \frac{\partial C(x, y, z, t)}{\partial y}\right|_{y=0}=\left.0 \frac{\partial C(x, y, z, t)}{\partial y}\right|_{x=L_{y}}=0, \\
& \left.\frac{\partial C(x, y, z, t)}{\partial z}\right|_{z=0}=\left.0 \frac{\partial C(x, y, z, t)}{\partial z}\right|_{x=L_{z}}=0, \mathrm{C}(\mathrm{x}, \mathrm{y}, \mathrm{z}, 0)=\mathrm{f}(\mathrm{x}, \mathrm{y}, \mathrm{z})
\end{aligned}
$$

The function $C(x, y, z, t)$ describes the distribution of dopant concentration in space and time; $T$ is the annealing temperature; parameter $D_{C}$ describes the diffusion coefficient of dopant. Let us approximate considered dependences of diffusion coefficient of dopant on different parameters by using the relation, which has been obtained by analysis [20]-[22]

$$
D_{C}=D_{L}(x, y, z, T)\left[1+\xi \frac{C^{\gamma}(x, y, z, t)}{P^{\gamma}(x, y, z, T)}\right]\left[1+\varsigma_{1} \frac{V(x, y, z, t)}{V^{*}}+\varsigma_{2} \frac{V^{2}(x, y, z, t)}{\left(V^{*}\right)^{2}}\right]
$$

The dependence $D_{L}(x, y, z, T)$ is the independent on any concentrations part of dopant diffusion coefficient. The dependence $P(x, y, z, T)$ is the dopant solubility limit. Value $\gamma \in[1]$, [3] is integer in the 
considered interval [20]. Dependences $V(x, y, z, t)$ is the radiation vacancies concentration distribution in space and time with equilibrium distribution $V^{*}$. The considered dependence of diffusion coefficient of dopant on concentration $C(x, y, z, t)$ has been discussed in [20]. It is known that diffusion doping did not leads to generation of radiation defects and $\zeta_{1}=\zeta_{2}=0$. We calculate distributions of radiation defects concentrations in space and time as solution of the equations, which are presented [21]-[23].

$$
\begin{aligned}
& \frac{\partial I(x, y, z, t)}{\partial t}=\frac{\partial}{\partial x}\left[D_{I}(x, y, z, T) \frac{\partial I(x, y, z, t)}{\partial x}\right]+\frac{\partial}{\partial y}\left[D_{I}(x, y, z, T) \frac{\partial I(x, y, z, t)}{\partial y}\right]-k_{I, I}(x, y, z, T) I^{2}(x, y, z, t)+ \\
& +\frac{\partial}{\partial z}\left[D_{I}(x, y, z, T) \frac{\partial I(x, y, z, t)}{\partial z}\right]-k_{I, V}(x, y, z, T) I(x, y, z, t) V(x, y, z, t) \\
& \frac{\partial V(x, y, z, t)}{\partial t}=\frac{\partial}{\partial x}\left[D_{V}(x, y, z, T) \frac{\partial V(x, y, z, t)}{\partial x}\right]+\frac{\partial}{\partial y}\left[D_{V}(x, y, z, T) \frac{\partial V(x, y, z, t)}{\partial y}\right]-k_{V, V}(x, y, z, T) V^{2}(x, y, z, t)+ \\
& +\frac{\partial}{\partial z}\left[D_{V}(x, y, z, T) \frac{\partial V(x, y, z, t)}{\partial z}\right]-k_{I, V}(x, y, z, T) I(x, y, z, t) V(x, y, z, t)
\end{aligned}
$$

Initial conditions and boundary conditions could be written as (5).

$$
\begin{aligned}
& \left.\frac{\partial \rho(x, y, z, t)}{\partial x}\right|_{x=0}=0,\left.\frac{\partial \rho(x, y, z, t)}{\partial x}\right|_{x=L_{x}}=0,\left.\frac{\partial \rho(x, y, z, t)}{\partial y}\right|_{y=0}=0,\left.\frac{\partial \rho(x, y, z, t)}{\partial y}\right|_{y=L_{y}}=0, \\
& \left.\frac{\partial \rho(x, y, z, t)}{\partial z}\right|_{z=0}=0,\left.\frac{\partial \rho(x, y, z, t)}{\partial z}\right|_{z=L_{z}}=0, \rho(\mathrm{x}, \mathrm{y}, \mathrm{z}, 0)=\mathrm{f}_{\rho}(\mathrm{x}, \mathrm{y}, \mathrm{z})
\end{aligned}
$$

Here are $\rho=I, V$. The considered function $I(x, y, z, t)$ describes distribution of radiation interstitials concentration in space and time with equilibrium distribution $I^{*}$; functions $D_{\rho}(x, y, z, T)$ describe dependences of point radiation defects diffusion coefficients on spatial coordinates and temperature; terms $V^{2}(x, y, z, t)$ and $I^{2}(x, y, z, t)$ describe generation of divacancies and diinterstitials, respectively; function $k_{I, V}(x, y, z, T)$ describes dependences of recombination parameter of point radiation defects on spatial coordinates and temperature; functions $k_{I, I}(x, y, z, T)$ and $k_{V, V}(x, y, z, T)$ describe dependences of parameters of point radiation defects simplest complexes generation on spatial coordinates and temperature. Now let us calculate spatio-temporal distributions of divacancies $\Phi_{V}(x, y, z, t)$ and diinterstitials $\Phi_{I}(x, y, z, t)$ concentrations by solving of the equations, which are presented [21]-[23]

$$
\begin{aligned}
& \frac{\partial \Phi_{I}(x, y, z, t)}{\partial t}=\frac{\partial}{\partial x}\left[D_{\Phi I}(x, y, z, T) \frac{\partial \Phi_{I}(x, y, z, t)}{\partial x}\right]+\frac{\partial}{\partial y}\left[D_{\Phi I}(x, y, z, T) \frac{\partial \Phi_{I}(x, y, z, t)}{\partial y}\right]+ \\
& \frac{\partial \Phi_{V}(x, y, z, t)}{\partial t}=\frac{\partial}{\partial x}\left[D_{\Phi V}(x, y, z, T) \frac{\partial \Phi_{V}(x, y, z, t)}{\partial x}\right]+\frac{\partial}{\partial y}\left[D_{\Phi V}(x, y, z, T) \frac{\partial \Phi_{V}(x, y, z, t)}{\partial y}\right]+ \\
& +\frac{\partial}{\partial z}\left[D_{\Phi V}(x, y, z, T) \frac{\partial \Phi_{V}(x, y, z, t)}{\partial z}\right]+k_{V, V}(x, y, z, T) V^{2}(x, y, z, t)-k_{V}(x, y, z, T) V(x, y, z, t)
\end{aligned}
$$

Initial conditions and boundary conditions could be written as (7).

$$
\begin{aligned}
& \left.\frac{\partial \Phi_{\rho}(x, y, z, t)}{\partial x}\right|_{x=0}=0,\left.\frac{\partial \Phi_{\rho}(x, y, z, t)}{\partial x}\right|_{x=L_{x}}=0,\left.\frac{\partial \Phi_{\rho}(x, y, z, t)}{\partial y}\right|_{y=0}=0,\left.\frac{\partial \Phi_{\rho}(x, y, z, t)}{\partial y}\right|_{y=L_{y}}=0, \\
& \left.\frac{\partial \Phi_{\rho}(x, y, z, t)}{\partial z}\right|_{z=0}=0,\left.\frac{\partial \Phi_{\rho}(x, y, z, t)}{\partial z}\right|_{z=L_{z}}=0, \Phi_{\mathrm{I}}(\mathrm{x}, \mathrm{y}, \mathrm{z}, 0)=\mathrm{f}_{\Phi \mathrm{I}}(\mathrm{x}, \mathrm{y}, \mathrm{z}), \Phi_{\mathrm{V}}(\mathrm{x}, \mathrm{y}, \mathrm{z}, 0)=\mathrm{f}_{\Phi \mathrm{V}}(\mathrm{x}, \mathrm{y}, \mathrm{z}) .
\end{aligned}
$$

Functions $D_{\Phi \rho}(x, y, z, T)$ describe dependences of diffusion coefficients of simplest complexes of point radiation defects on spatial coordinates and temperature; functions $k_{I}(x, y, z, T)$ and $k_{V}(x, y, z, T)$ describe 
dependences of parameters of decay of the considered complexes on spatial coordinates and temperature. We obtain spatio-temporal distributions of point radiation defects concentrations concentrations framework approach, which has been recently elaborated [19]. Framework the approach we transform diffusion coefficients approximations to the next form: $D_{\rho}(x, y, z, T)=D_{0 \rho}\left[1+\varepsilon_{\rho} g_{\rho}(x, y, z, T)\right]$. Here $D_{0 \rho}$ describe the diffusion coefficients average values, $0 \leq \varepsilon_{\rho}<1,\left|g_{\rho}(x, y, z, T)\right| \leq 1, \rho=I, V$. The same transformation we used for approximations of generation of their complexes parameters and recombination of point defects: $k_{I, V}(x, y, z$, $T)=k_{0 I, V}\left[1+\varepsilon_{I, V} g_{I, V}(x, y, z, T)\right], k_{I, I}(x, y, z, T)=k_{0 I, I}\left[1+\varepsilon_{I, I} \quad g_{I, I}(x, y, z, T)\right]$ and $k_{V, V}(x, y, z, T)=k_{0 V, V}\left[1+\varepsilon_{V, V} \quad g_{V, V}(x, y, z, T)\right]$. Here $k_{0 \rho 1, \rho_{2}}$ are the averaged values of the considered parameters, $0 \leq \varepsilon_{I, V}<1,0 \leq \varepsilon_{I, I}<1,0 \leq \varepsilon_{V, V}<1, \mid g_{I, V}(x, y, z$, $T)|\leq 1,| g_{I, I}(x, y, z, T)|\leq 1,| g_{V, V}(x, y, z, T) \mid \leq 1$. Let us introduce the following dimensionless variables: $\chi=x / L_{x}$, $\vartheta=\sqrt{D_{0 I} D_{0 V}} t / L^{2}, \tilde{I}(x, y, z, t)=I(x, y, z, t) / I^{*}, \tilde{V}(x, y, z, t)=V(x, y, z, t) / V^{*}, \omega=L^{2} k_{0 I, V} / \sqrt{D_{0 I} D_{0 V}}$ , $\eta=y / L_{y}, \Omega_{\rho}=L^{2} k_{0 \rho, \rho} / \sqrt{D_{0 I} D_{0 V}}, \phi=z / L_{z}$. After using of these variables we obtain (4) and conditions (5) in the following form

$$
\begin{gathered}
\frac{\partial \tilde{I}(\chi, \eta, \phi, \vartheta)}{\partial \vartheta}=\frac{D_{0 I}}{\sqrt{D_{0 I} D_{0 V}}} \frac{\partial}{\partial \chi}\left\{\left[1+\varepsilon_{I} g_{I}(\chi, \eta, \phi, T)\right] \frac{\partial \tilde{I}(\chi, \eta, \phi, \vartheta)}{\partial \chi}\right\}+\frac{\partial}{\partial \eta}\left\{\left[1+\varepsilon_{I} g_{I}(\chi, \eta, \phi, T)\right] \frac{\partial \tilde{I}(\chi, \eta, \phi, \vartheta)}{\partial \eta}\right\} \times \\
\times \frac{D_{0 I}}{\sqrt{D_{0 I} D_{0 V}}}+\frac{D_{0 I}}{\sqrt{D_{0 I} D_{0 V}}} \frac{\partial}{\partial \phi}\left\{\left[1+\varepsilon_{I} g_{I}(\chi, \eta, \phi, T)\right] \frac{\partial \tilde{I}(\chi, \eta, \phi, \vartheta)}{\partial \phi}\right\}-\omega\left[1+\varepsilon_{I, V} g_{I, V}(\chi, \eta, \phi, T)\right] \tilde{I}(\chi, \eta, \phi, \vartheta) \times \\
\times \tilde{V}(\chi, \eta, \phi, \vartheta)-\Omega_{I}\left[1+\varepsilon_{I, I} g_{I, I}(\chi, \eta, \phi, T)\right] \tilde{I}^{2}(\chi, \eta, \phi, \vartheta) \\
\left.\frac{\partial \tilde{\rho}(\chi, \eta, \phi, \vartheta)}{\partial \chi}\right|_{\chi=0}=0,\left.\frac{\partial \tilde{\rho}(\chi, \eta, \phi, \vartheta)}{\partial \chi}\right|_{\chi=1}=0,\left.\frac{\partial \tilde{\rho}(\chi, \eta, \phi, \vartheta)}{\partial \eta}\right|_{\eta=0}=0,\left.\frac{\partial \tilde{\rho}(\chi, \eta, \phi, \vartheta)}{\partial \eta}\right|_{\eta=1}=0 \\
\left.\frac{\partial \tilde{\rho}(\chi, \eta, \phi, \vartheta)}{\partial \phi}\right|_{\phi=0}=0,\left.\frac{\partial \tilde{\rho}(\chi, \eta, \phi, \vartheta)}{\partial \phi}\right|_{\phi=1}=0, \tilde{\rho}(\chi, \eta, \phi, \vartheta)=\frac{f_{\rho}(\chi, \eta, \phi, \vartheta)}{\rho^{*}}
\end{gathered}
$$

We calculate solutions of (8) with account conditions (9) by using approach, which was introduced recently [18]. Framework the approach we determine the required solution as

$$
\tilde{\rho}(\chi, \eta, \phi, \vartheta)=\sum_{i=0}^{\infty} \varepsilon_{\rho}^{i} \sum_{j=0}^{\infty} \omega^{j} \sum_{k=0}^{\infty} \Omega_{\rho}^{k} \tilde{\rho}_{i j k}(\chi, \eta, \phi, \vartheta)
$$

After substitution of the above series (10) into recently considered (8) with conditions (9) leads to obtaining of equations for the zero-order approximations of point radiation defects concentrations $\tilde{I}_{000}(\chi, \eta, \phi, \vartheta)$ and $\tilde{V}_{000}(\chi, \eta, \phi, \vartheta)$. Also we obtain corrections for them $\tilde{I}_{i j k}(\chi, \eta, \phi, \vartheta)$ and $\tilde{V}_{i j k}(\chi, \eta, \phi, \vartheta), i \geq 1, j \geq 1, k \geq 1$ based on the substitution. We present the obtained equations in Appendix in the end of the paper. We solved the obtained equations by Fourier approach in the standard form [24], [25]. We present the obtained solutions in the Appendix in the end of the paper. Now let us calculate of point radiation defects of simplest complexes distributions of concentrations in space and time. Let us transform diffusion coefficient's approximations to the next form: $D_{\Phi \rho}(x, y, z, T)=D_{0 \Phi \rho}[1+\varepsilon \Phi \rho g \Phi \rho(x, y, z, T)]$. Here $D_{0} \Phi \rho$ are the averaged means of the considered diffusion coefficients. After this transformation we obtain (6) in this form

$$
\begin{aligned}
& \frac{\partial \Phi_{I}(x, y, z, t)}{\partial t}=D_{0 \Phi I} \frac{\partial}{\partial x}\left\{\left[1+\varepsilon_{\Phi I} g_{\Phi I}(x, y, z, T)\right] \frac{\partial \Phi_{I}(x, y, z, t)}{\partial x}\right\}+k_{I, I}(x, y, z, T) I^{2}(x, y, z, t)-k_{I}(x, y, z, T) \times \\
& \times I(x, y, z, t)+D_{0 \Phi I} \frac{\partial}{\partial y}\left\{\left[1+\varepsilon_{\Phi I} g_{\Phi I}(x, y, z, T)\right] \frac{\partial \Phi_{I}(x, y, z, t)}{\partial y}\right\}+D_{0 \Phi I} \frac{\partial}{\partial z}\left\{\left[1+\varepsilon_{\Phi I} g_{\Phi I}(x, y, z, T)\right] \frac{\partial \Phi_{I}(x, y, z, t)}{\partial z}\right\} \\
& \frac{\partial \Phi_{V}(x, y, z, t)}{\partial t}=D_{0 \Phi V} \frac{\partial}{\partial x}\left\{\left[1+\varepsilon_{\Phi V} g_{\Phi V}(x, y, z, T)\right] \frac{\partial \Phi_{V}(x, y, z, t)}{\partial x}\right\}+k_{I, I}(x, y, z, T) I^{2}(x, y, z, t)-k_{I}(x, y, z, T) \times \\
& \times I(x, y, z, t)+D_{0 \Phi V} \frac{\partial}{\partial y}\left\{\left[1+\varepsilon_{\Phi V} g_{\Phi V}(x, y, z, T)\right] \frac{\partial \Phi_{V}(x, y, z, t)}{\partial y}\right\}+D_{0 \Phi V} \frac{\partial}{\partial z}\left\{\left[1+\varepsilon_{\Phi V} g_{\Phi V}(x, y, z, T)\right] \frac{\partial \Phi_{V}(x, y, z, t)}{\partial z}\right\}
\end{aligned}
$$


After considered transformation we will determine solution of obtained equations in the following form (11).

$$
\Phi_{\rho}(x, y, z, t)=\sum_{i=0}^{\infty} \varepsilon_{\Phi \rho}^{i} \Phi_{\rho i}(x, y, z, t)
$$

Substitution of the considered series into above equations and conditions for them leads to obtaining equations for functions $\Phi_{I i}(x, y, z, t)$ and $\Phi_{V i}(x, y, z, t)$ (for $\left.i \geq 0\right)$ and conditions for them. We present the obtained equations and conditions in the Appendix in the end of this paper. We solved the obtained equations by Fourier approach in the standard form [24], [25]. We present the obtained solutions in the Appendix in the end of the paper.

Now let us determine spatio-temporal distribution of dopant concentration by using the same approach, which we used for solution of equations of radiation defects. In this situation we again transform approximation of diffusion coefficient (now diffusion coefficient of dopant) to the same form as for diffusion coefficient of radiation defects: $D_{L}(x, y, z, T)=D_{0 L}\left[1+\varepsilon_{L} g_{L}(x, y, z, T)\right]$. Here $D_{0 L}$ is the averages mean of diffusion coefficient of dopant, $0 \leq \varepsilon_{L}<1,\left|g_{L}(x, y, z, T)\right| \leq 1$. Now let us to solve (1) as the following form:

$$
C(x, y, z, t)=\sum_{i=0}^{\infty} \varepsilon_{L}^{i} \sum_{j=1}^{\infty} \xi^{j} C_{i j}(x, y, z, t)
$$

Now we take into account this series into (1) and relations (2). After this accounting we obtain equations for all functions $C_{i j}(x, y, z, t)$ (for $i \geq 0, j \geq 0$ ) and conditions for them. The obtained equations and conditions for them are presented in the Appendix. We solved the obtained equations by Fourier approach in the standard form [24], [25] and present these solutions in the Appendix in the end of paper.

In this paper we analyzed spatio-temporal distributions of radiation defects and infused or implanted dopant concentrations. To make the analysis we used the second-order approximations of these concentrations on all parameters, which were used framework obtained solutions. Usually, the considered approximation is enough good approximation to obtain some quantitative results and make some qualitative analysis. All obtained analytical results were checked by comparison with results of numerical simulation.

\section{DISCUSSION}

Now we make analysis of spatio-temporal distributions of dopant with account redistribution of radiation defects. We present on Figure 2 typical distributions of dopant concentration in space near interface between layers of heterostructures. We obtain the considered distributions for the following case: diffusion coefficient of dopant in doped area is larger, than the same coefficient in the nearest undoped areas.

Under the condition the Figure 2 shows increasing of compactness of dopant distributions framework the field-effect heterotransistors (increasing of number of curves on these figures describes larger difference between magnitudes of coefficients of diffusion of dopant in layers of the considered heterostructure). At the same time one can find, that homogeneity of distribution of dopant concentration increases. Vice versa relation between values of diffusion coefficients of dopant leads to vice versa result see Figure 3, increasing of number of curves on these figures describes larger difference between magnitudes of coefficients of diffusion of dopant in layers of the considered heterostructure).

It is necessary to note, that using the considered approach of manufacturing of field-effect heterotransistors leads to necessity optimization of value of annealing time of the considered dopant. We optimize the annealing time by using the criterion, which has been recently introduced [26]-[32]. To make the optimization we approximate real distribution of concentration of dopant by step-wise function $\psi(x, y, z)$. These approximations are presented on Figure 4. The considered value of optimal annealing time we obtained by minimization of mean-squared error

$$
U=\frac{1}{L_{x} L_{y} L_{z}} \int_{0}^{L_{x}} \int_{0}^{L_{y} L_{z}} \int_{0}[C(x, y, z, \Theta)-\psi(x, y, z)] d z d y d x
$$




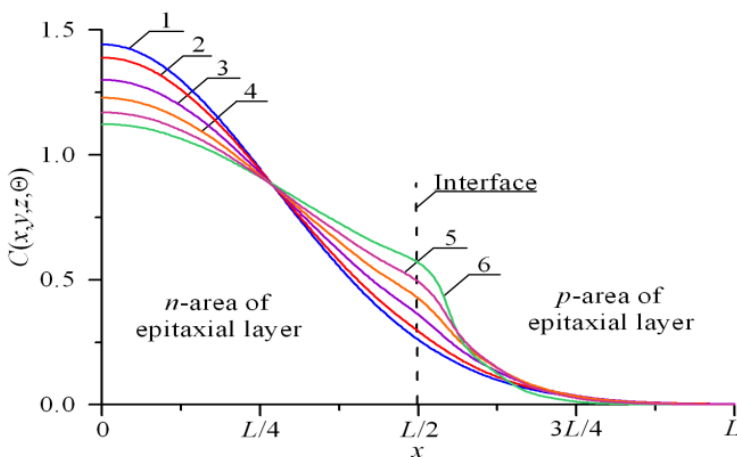

(a)

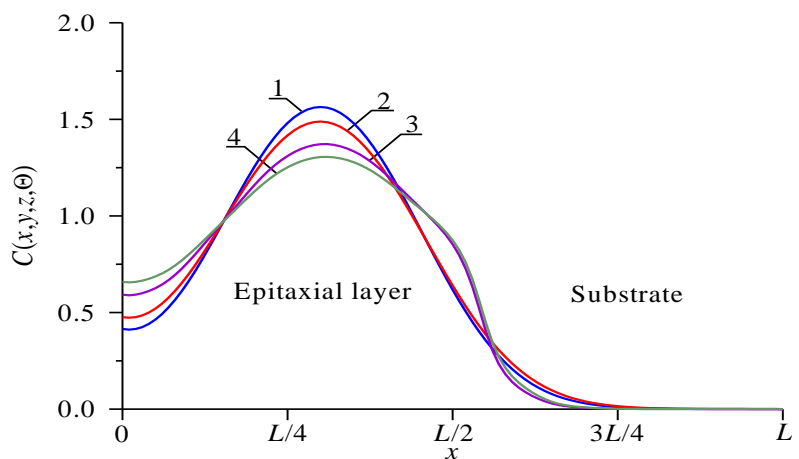

(b)

Figure 2. These figures are (a) Distributions of infused dopant concentration, and (b) Distributions of implanted dopant concentration. Curve 1 and curve 3 describe distribution of dopant at time of annealing $\Theta=$

$0.0048\left(L_{x}^{2}+L_{y}^{2}+L_{z}^{2}\right) / D_{0}$. Curve 2 and curve 4 describe distribution of dopant at time of annealing $\Theta=$

$$
0.0057\left(L_{x}^{2}+L_{y}^{2}+L_{z}^{2}\right) / D_{0}
$$

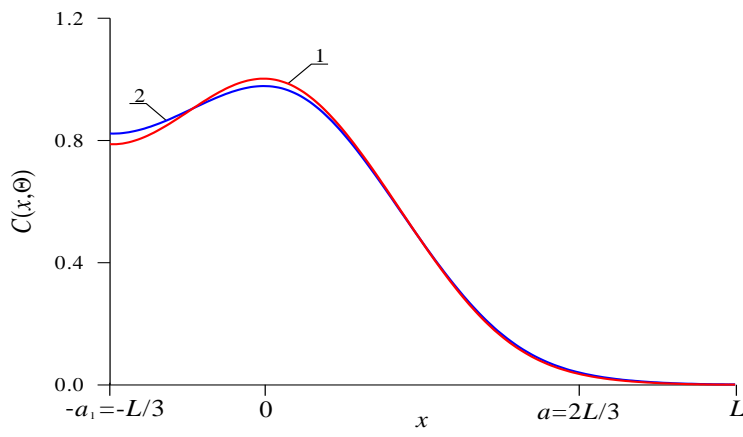

(a)

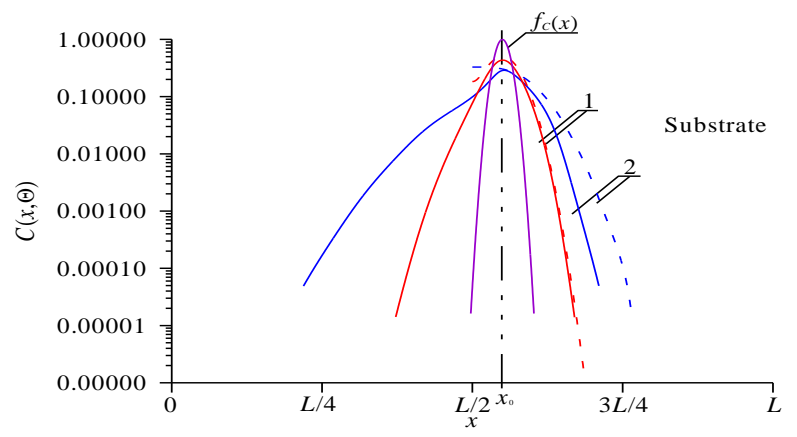

(b)

Figure 3. These figures are (a) Distributions of infused dopant concentration, and (b) Distributions of implanted dopant concentration. Solid lines describe distributions dopant in heterostructure, which includes into itself two layers: substrate and epitaxial layer. Dushed lines describe distributions dopant in a single layer

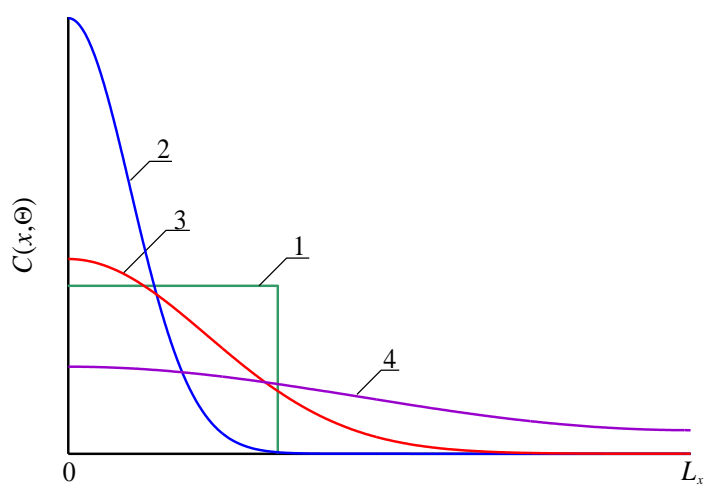

(a)

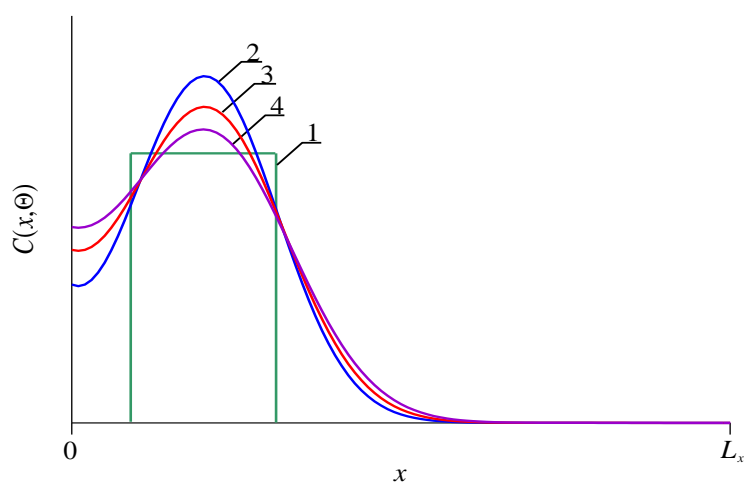

(b)

Figure 4. These figures are (a) Infused dopant distributions. Curve 1 describes idealized stepwise distribution. Curve 2, curve 3 and curve 4 describe actually existing distributions of concentration of dopant at different means of time of annealing, and (b) Implanted dopant distributions. Curve 1 describes idealized stepwise distribution. Curve 2, curve 3 and curve 4 describe actually existing distributions of concentration of dopant at different means of time of annealing 
Dependences of optimal means of time of annealing on different parameters were presented on Figure 5. Standard procedure after ion implantation is annealing of generation during the implantation radiation defects. During ideal annealing of defects dopant should achieves nearest interface between layers of considered heterostructure. If the considered dopant does not achieve the interface the annealing, then it is attracted an interests additional annealing of dopant. In this situation annealing time from Figure 5(b) is smaller, than analogous time from Figure 5(a).

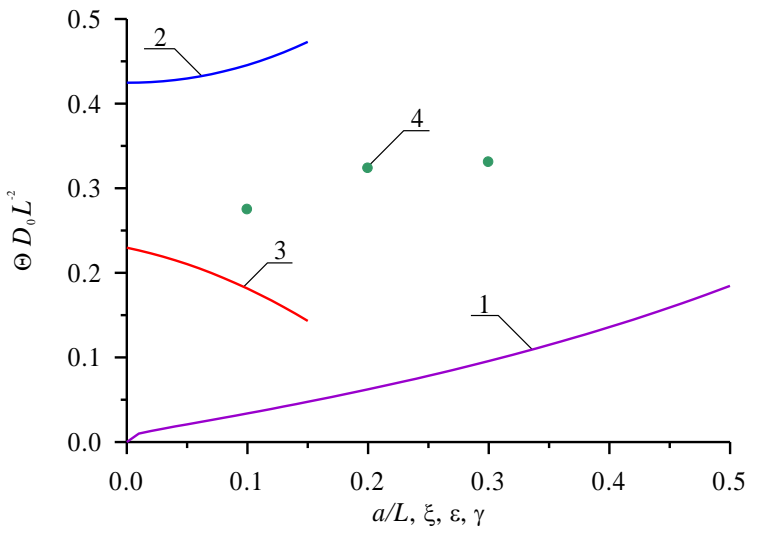

(a)

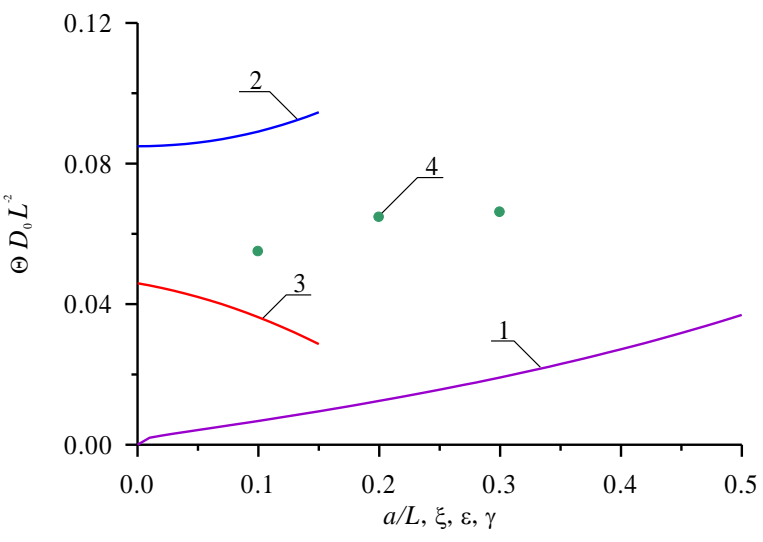

(b)

Figure 5. These figures are; (a) Dimensionless optimized time of annealing of infused dopant as a function of several parameters. Curve 1 as a function of dimensionless optimized time of annealing on relation a/L and $\xi$ $=\gamma=0$ at the equal values to each other of dopant diffusion coefficient in all materials of heterostructure.

Curve 2 as a function of dimensionless optimized time of annealing of the parameter $\varepsilon$ at $\mathrm{a} / \mathrm{L}=1 / 2$ and $\xi=\gamma=$

0 . Curve 3 as a function of dimensionless optimized time of annealing on parameter $\xi$ at $\mathrm{a} / \mathrm{L}=1 / 2$ and $\varepsilon=\gamma=0$.

Curve 4 as a function of dimensionless optimized time of annealing of parameter $\gamma$ at $\mathrm{a} / \mathrm{L}=1 / 2$ and $\varepsilon=\xi=0$.

(b) Dimensionless optimal annealing time of implanted dopant as a function of several parameters. Curve 1 describes the dependence of the annealing time on the relation $\mathrm{a} / \mathrm{L}$ and $\xi=\gamma=0$ for equal to each other values of dopant diffusion coefficient in all parts of heterostructure. Curve 2 describes the dependence of the annealing time on value of parameter $\varepsilon$ for $\mathrm{a} / \mathrm{L}=1 / 2$ and $\xi=\gamma=0$. Curve 3 describes the dependence of the annealing time on value of parameter $\xi$ for $\mathrm{a} / \mathrm{L}=1 / 2$ and $\varepsilon=\gamma=0$. Curve 4 describes the dependence of the annealing time on value of parameter $\gamma$ for $\mathrm{a} / \mathrm{L}=1 / 2$ and $\varepsilon=\xi=0$

In this section, it is explained the results of research and at the same time is given the comprehensive discussion. Results can be presented in figures, graphs, tables, and others that make the reader understand easily [2], [5]. The discussion can be made in several sub-chapters.

\section{CONCLUSION}

Based on modelling of redistribution of implanted and infused dopants in heterostructures during formation of field-effect heterotransistors in switched-capacitor step-down DC-DC converter we formulate recommendations to modify technological process for decreasing of dimensions of the considered transistors and with simultaneous increasing of their de

Nsity. We also formulate several recommendations for decreasing of mismatch-induced stress in heterostructures. As an accompany result of this paper we introduce an analytical approach for prognosis of mass and heat transport during manufacturing of elements of integrated circuits.

\section{APPENDIX}

Functions $\tilde{I}_{i j k}(\chi, \eta, \phi, \vartheta)$ and $\tilde{V}_{i j k}(\chi, \eta, \phi, \vartheta)$ described by the following equations for any values of indexes $i \geq 0, j \geq 0, k \geq 0$ 


$$
\begin{aligned}
& \frac{\partial \tilde{I}_{000}(\chi, \eta, \phi, \vartheta)}{\partial \vartheta}=\sqrt{\frac{D_{0 I}}{D_{0 V}}}\left[\frac{\partial^{2} \tilde{I}_{000}(\chi, \eta, \phi, \vartheta)}{\partial \chi^{2}}+\frac{\partial^{2} \tilde{I}_{000}(\chi, \eta, \phi, \vartheta)}{\partial \eta^{2}}+\frac{\partial^{2} \tilde{I}_{000}(\chi, \eta, \phi, \vartheta)}{\partial \phi^{2}}\right] \\
& \frac{\partial \tilde{V}_{000}(\chi, \eta, \phi, \vartheta)}{\partial \vartheta}=\sqrt{\frac{D_{0 V}}{D_{0 I}}}\left[\frac{\partial^{2} \tilde{V}_{000}(\chi, \eta, \phi, \vartheta)}{\partial \chi^{2}}+\frac{\partial^{2} \tilde{V}_{000}(\chi, \eta, \phi, \vartheta)}{\partial \eta^{2}}+\frac{\partial^{2} \tilde{V}_{000}(\chi, \eta, \phi, \vartheta)}{\partial \phi^{2}}\right] ; \\
& \frac{\partial \tilde{I}_{i 00}(\chi, \vartheta)}{\partial \vartheta}=\sqrt{\frac{D_{0 I}}{D_{0 V}}}\left[\frac{\partial^{2} \tilde{I}_{i 00}(\chi, \eta, \phi, \vartheta)}{\partial \chi^{2}}+\frac{\partial^{2} \tilde{I}_{i 00}(\chi, \eta, \phi, \vartheta)}{\partial \eta^{2}}+\frac{\partial^{2} \tilde{I}_{i 00}(\chi, \eta, \phi, \vartheta)}{\partial \phi^{2}}\right]+ \\
& +\sqrt{\frac{D_{0 I}}{D_{0 V}}}\left\{\frac{\partial}{\partial \chi}\left[g_{I}(\chi, \eta, \phi, T) \frac{\partial \tilde{I}_{i-100}(\chi, \eta, \phi, \vartheta)}{\partial \chi}\right]+\frac{\partial}{\partial \eta}\left[g_{I}(\chi, \eta, \phi, T) \frac{\partial \tilde{I}_{i-100}(\chi, \eta, \phi, \vartheta)}{\partial \eta}\right]+\right. \\
& \left.+\frac{\partial}{\partial \phi}\left[g_{I}(\chi, \eta, \phi, T) \frac{\partial \tilde{I}_{i-100}(\chi, \eta, \phi, \vartheta)}{\partial \phi}\right]\right\}, i \geq 1,
\end{aligned}
$$$$
\frac{\partial \tilde{V}_{i 00}(\chi, \vartheta)}{\partial \vartheta}=\sqrt{\frac{D_{0 V}}{D_{0 I}}}\left[\frac{\partial^{2} \tilde{V}_{i 00}(\chi, \eta, \phi, \vartheta)}{\partial \chi^{2}}+\frac{\partial^{2} \tilde{V}_{i 00}(\chi, \eta, \phi, \vartheta)}{\partial \eta^{2}}+\frac{\partial^{2} \tilde{V}_{i 00}(\chi, \eta, \phi, \vartheta)}{\partial \phi^{2}}\right]+\sqrt{\frac{D_{0 V}}{D_{0 I}}} \frac{\partial}{\partial \chi}\left[g_{V}(\chi, \eta, \phi, T) \frac{\partial \tilde{V}_{i-100}(\chi, \eta, \phi, \vartheta)}{\partial \chi}\right]+
$$$$
+\sqrt{\frac{D_{0 V}}{D_{0 I}}} \frac{\partial}{\partial \eta}\left[g_{V}(\chi, \eta, \phi, T) \frac{\partial \tilde{V}_{i-100}(\chi, \eta, \phi, \vartheta)}{\partial \eta}\right]+\sqrt{\frac{D_{0 V}}{D_{0 I}}} \frac{\partial}{\partial \phi}\left[g_{V}(\chi, \eta, \phi, T) \frac{\partial \tilde{V}_{i-100}(\chi, \eta, \phi, \vartheta)}{\partial \phi}\right], i \geq 1,
$$$$
\frac{\partial \tilde{I}_{010}(\chi, \eta, \phi, \vartheta)}{\partial \vartheta}=\sqrt{\frac{D_{0 I}}{D_{0 V}}}\left[\frac{\partial^{2} \tilde{I}_{010}(\chi, \eta, \phi, \vartheta)}{\partial \chi^{2}}+\frac{\partial^{2} \tilde{I}_{010}(\chi, \eta, \phi, \vartheta)}{\partial \eta^{2}}+\frac{\partial^{2} \tilde{I}_{010}(\chi, \eta, \phi, \vartheta)}{\partial \phi^{2}}\right]-
$$$$
-\left[1+\varepsilon_{I, V} g_{I, V}(\chi, \eta, \phi, T)\right] \tilde{I}_{000}(\chi, \eta, \phi, \vartheta) \tilde{V}_{000}(\chi, \eta, \phi, \vartheta)
$$$$
\frac{\partial \tilde{V}_{010}(\chi, \eta, \phi, \vartheta)}{\partial \vartheta}=\sqrt{\frac{D_{0 V}}{D_{0 I}}}\left[\frac{\partial^{2} \tilde{V}_{010}(\chi, \eta, \phi, \vartheta)}{\partial \chi^{2}}+\frac{\partial^{2} \tilde{V}_{010}(\chi, \eta, \phi, \vartheta)}{\partial \eta^{2}}+\frac{\partial^{2} \tilde{V}_{010}(\chi, \eta, \phi, \vartheta)}{\partial \phi^{2}}\right]-
$$$$
-\left[1+\varepsilon_{I, V} g_{I, V}(\chi, \eta, \phi, T)\right] \tilde{I}_{000}(\chi, \eta, \phi, \vartheta) \tilde{V}_{000}(\chi, \eta, \phi, \vartheta)
$$$$
\frac{\partial \tilde{I}_{020}(\chi, \eta, \phi, \vartheta)}{\partial \vartheta}=\sqrt{\frac{D_{0 I}}{D_{0 V}}}\left[\frac{\partial^{2} \widetilde{I}_{020}(\chi, \eta, \phi, \vartheta)}{\partial \chi^{2}}+\frac{\partial^{2} \tilde{I}_{020}(\chi, \eta, \phi, \vartheta)}{\partial \eta^{2}}+\frac{\partial^{2} \tilde{I}_{020}(\chi, \eta, \phi, \vartheta)}{\partial \phi^{2}}\right]-
$$$$
-\left[1+\varepsilon_{I, V} g_{I, V}(\chi, \eta, \phi, T)\right]\left[\tilde{I}_{010}(\chi, \eta, \phi, \vartheta) \tilde{V}_{000}(\chi, \eta, \phi, \vartheta)+\tilde{I}_{000}(\chi, \eta, \phi, \vartheta) \tilde{V}_{010}(\chi, \eta, \phi, \vartheta)\right]
$$$$
\frac{\partial \tilde{V}_{020}(\chi, \eta, \phi, \vartheta)}{\partial \vartheta}=\sqrt{\frac{D_{0 I}}{D_{0 V}}}\left[\frac{\partial^{2} \tilde{V}_{020}(\chi, \eta, \phi, \vartheta)}{\partial \chi^{2}}+\frac{\partial^{2} \tilde{V}_{020}(\chi, \eta, \phi, \vartheta)}{\partial \eta^{2}}+\frac{\partial^{2} \tilde{V}_{020}(\chi, \eta, \phi, \vartheta)}{\partial \phi^{2}}\right]-
$$$$
-\left[1+\varepsilon_{I, V} g_{I, V}(\chi, \eta, \phi, T)\right]\left[\tilde{I}_{010}(\chi, \eta, \phi, \vartheta) \tilde{V}_{000}(\chi, \eta, \phi, \vartheta)+\tilde{I}_{000}(\chi, \eta, \phi, \vartheta) \tilde{V}_{010}(\chi, \eta, \phi, \vartheta)\right]
$$ 


$$
\begin{aligned}
& \frac{\partial \tilde{I}_{001}(\chi, \eta, \phi, \vartheta)}{\partial \vartheta}=\sqrt{\frac{D_{0 I}}{D_{0 V}}}\left[\frac{\partial^{2} \widetilde{I}_{001}(\chi, \eta, \phi, \vartheta)}{\partial \chi^{2}}+\frac{\partial^{2} \widetilde{I}_{001}(\chi, \eta, \phi, \vartheta)}{\partial \eta^{2}}+\frac{\partial^{2} \widetilde{I}_{001}(\chi, \eta, \phi, \vartheta)}{\partial \phi^{2}}\right]- \\
& -\left[1+\varepsilon_{I, I} g_{I, I}(\chi, \eta, \phi, T)\right] \tilde{I}_{000}^{2}(\chi, \eta, \phi, \vartheta) \\
& \frac{\partial \tilde{V}_{001}(\chi, \eta, \phi, \vartheta)}{\partial \vartheta}=\sqrt{\frac{D_{0 V}}{D_{0 I}}}\left[\frac{\partial^{2} \tilde{V}_{001}(\chi, \eta, \phi, \vartheta)}{\partial \chi^{2}}+\frac{\partial^{2} \tilde{V}_{001}(\chi, \eta, \phi, \vartheta)}{\partial \eta^{2}}+\frac{\partial^{2} \tilde{V}_{001}(\chi, \eta, \phi, \vartheta)}{\partial \phi^{2}}\right]- \\
& -\left[1+\varepsilon_{I, I} g_{I, I}(\chi, \eta, \phi, T)\right] \tilde{V}_{000}^{2}(\chi, \eta, \phi, \vartheta) \\
& \frac{\partial \tilde{I}_{110}(\chi, \eta, \phi, \vartheta)}{\partial \vartheta}=\sqrt{\frac{D_{0 I}}{D_{0 V}}}\left[\frac{\partial^{2} \tilde{I}_{110}(\chi, \eta, \phi, \vartheta)}{\partial \chi^{2}}+\frac{\partial^{2} \tilde{I}_{110}(\chi, \eta, \phi, \vartheta)}{\partial \eta^{2}}+\frac{\partial^{2} \tilde{I}_{110}(\chi, \eta, \phi, \vartheta)}{\partial \phi^{2}}\right]+\sqrt{\frac{D_{0 I}}{D_{0 V}}}\left\{\frac { \partial } { \partial \chi } \left[g_{I}(\chi, \eta, \phi, T) \times\right.\right. \\
& \left.\left.\times \frac{\partial \tilde{I}_{010}(\chi, \eta, \phi, \vartheta)}{\partial \chi}\right]+\frac{\partial}{\partial \eta}\left[g_{I}(\chi, \eta, \phi, T) \frac{\partial \tilde{I}_{010}(\chi, \eta, \phi, \vartheta)}{\partial \eta}\right]+\frac{\partial}{\partial \phi}\left[g_{I}(\chi, \eta, \phi, T) \frac{\partial \tilde{I}_{010}(\chi, \eta, \phi, \vartheta)}{\partial \phi}\right]\right\}- \\
& -\left[1+\varepsilon_{I, I} g_{I, I}(\chi, \eta, \phi, T)\right]\left[\tilde{I}_{100}(\chi, \eta, \phi, \vartheta) \tilde{V}_{000}(\chi, \eta, \phi, \vartheta)+\tilde{I}_{000}(\chi, \eta, \phi, \vartheta) \tilde{V}_{100}(\chi, \eta, \phi, \vartheta)\right] \\
& \frac{\partial \tilde{V}_{110}(\chi, \eta, \phi, \vartheta)}{\partial \vartheta}=\sqrt{\frac{D_{0 V}}{D_{0 I}}}\left[\frac{\partial^{2} \tilde{V}_{110}(\chi, \eta, \phi, \vartheta)}{\partial \chi^{2}}+\frac{\partial^{2} \tilde{V}_{110}(\chi, \eta, \phi, \vartheta)}{\partial \eta^{2}}+\frac{\partial^{2} \tilde{V}_{110}(\chi, \eta, \phi, \vartheta)}{\partial \phi^{2}}\right]+\sqrt{\frac{D_{0 V}}{D_{0 I}}}\left\{\frac { \partial } { \partial \chi } \left[g_{V}(\chi, \eta, \phi, T) \times\right.\right. \\
& \left.\left.\times \frac{\partial \tilde{V}_{010}(\chi, \eta, \phi, \vartheta)}{\partial \chi}\right]+\frac{\partial}{\partial \eta}\left[g_{V}(\chi, \eta, \phi, T) \frac{\partial \tilde{V}_{010}(\chi, \eta, \phi, \vartheta)}{\partial \eta}\right]+\frac{\partial}{\partial \phi}\left[g_{V}(\chi, \eta, \phi, T) \frac{\partial \tilde{V}_{010}(\chi, \eta, \phi, \vartheta)}{\partial \phi}\right]\right\}- \\
& -\left[1+\varepsilon_{V, V} g_{V, V}(\chi, \eta, \phi, T)\right]\left[\tilde{V}_{100}(\chi, \eta, \phi, \vartheta) \tilde{I}_{000}(\chi, \eta, \phi, \vartheta)+\tilde{V}_{000}(\chi, \eta, \phi, \vartheta) \tilde{I}_{100}(\chi, \eta, \phi, \vartheta)\right] \\
& \frac{\partial \tilde{I}_{002}(\chi, \eta, \phi, \vartheta)}{\partial \vartheta}=\sqrt{\frac{D_{0 I}}{D_{0 V}}}\left[\frac{\partial^{2} \tilde{I}_{002}(\chi, \eta, \phi, \vartheta)}{\partial \chi^{2}}+\frac{\partial^{2} \tilde{I}_{002}(\chi, \eta, \phi, \vartheta)}{\partial \eta^{2}}+\frac{\partial^{2} \tilde{I}_{002}(\chi, \eta, \phi, \vartheta)}{\partial \phi^{2}}\right]- \\
& -\left[1+\varepsilon_{I, I} g_{I, I}(\chi, \eta, \phi, T)\right] \tilde{I}_{001}(\chi, \eta, \phi, \vartheta) \tilde{I}_{000}(\chi, \eta, \phi, \vartheta) \\
& \frac{\partial \tilde{V}_{002}(\chi, \eta, \phi, \vartheta)}{\partial \vartheta}=\sqrt{\frac{D_{0 V}}{D_{0 I}}}\left[\frac{\partial^{2} \tilde{V}_{002}(\chi, \eta, \phi, \vartheta)}{\partial \chi^{2}}+\frac{\partial^{2} \tilde{V}_{002}(\chi, \eta, \phi, \vartheta)}{\partial \eta^{2}}+\frac{\partial^{2} \tilde{V}_{002}(\chi, \eta, \phi, \vartheta)}{\partial \phi^{2}}\right]- \\
& -\left[1+\varepsilon_{V, V} g_{V, V}(\chi, \eta, \phi, E)\right] \tilde{V}_{001}(\chi, \eta, \phi, \vartheta) \tilde{V}_{000}(\chi, \eta, \phi, \vartheta) \\
& \frac{\partial \tilde{I}_{101}(\chi, \eta, \phi, \vartheta)}{\partial \vartheta}=\sqrt{\frac{D_{0 I}}{D_{0 V}}}\left[\frac{\partial^{2} \tilde{I}_{101}(\chi, \eta, \phi, \vartheta)}{\partial \chi^{2}}+\frac{\partial^{2} \tilde{I}_{101}(\chi, \eta, \phi, \vartheta)}{\partial \eta^{2}}+\frac{\partial^{2} \tilde{I}_{101}(\chi, \eta, \phi, \vartheta)}{\partial \phi^{2}}\right]+ \\
& +\sqrt{\frac{D_{0 I}}{D_{0 V}}}\left\{\frac{\partial}{\partial \chi}\left[g_{I}(\chi, \eta, \phi, T) \frac{\partial \tilde{I}_{001}(\chi, \eta, \phi, \vartheta)}{\partial \chi}\right]+\frac{\partial}{\partial \eta}\left[g_{I}(\chi, \eta, \phi, T) \frac{\partial \tilde{I}_{001}(\chi, \eta, \phi, \vartheta)}{\partial \eta}\right]+\right.
\end{aligned}
$$


$\left.+\frac{\partial}{\partial \phi}\left[g_{I}(\chi, \eta, \phi, T) \frac{\partial \tilde{I}_{001}(\chi, \eta, \phi, \vartheta)}{\partial \phi}\right]\right\}-\left[1+\varepsilon_{I} g_{I}(\chi, \eta, \phi, T)\right] \tilde{I}_{100}(\chi, \eta, \phi, \vartheta) \tilde{V}_{000}(\chi, \eta, \phi, \vartheta)$

$\frac{\partial \tilde{V}_{101}(\chi, \eta, \phi, \vartheta)}{\partial \vartheta}=\sqrt{\frac{D_{0 V}}{D_{0 I}}}\left[\frac{\partial^{2} \tilde{V}_{101}(\chi, \eta, \phi, \vartheta)}{\partial \chi^{2}}+\frac{\partial^{2} \tilde{V}_{101}(\chi, \eta, \phi, \vartheta)}{\partial \eta^{2}}+\frac{\partial^{2} \tilde{V}_{101}(\chi, \eta, \phi, \vartheta)}{\partial \phi^{2}}\right]+$

$+\sqrt{\frac{D_{0 V}}{D_{0 I}}}\left\{\frac{\partial}{\partial \chi}\left[g_{V}(\chi, \eta, \phi, T) \frac{\partial \tilde{V}_{001}(\chi, \eta, \phi, \vartheta)}{\partial \chi}\right]+\frac{\partial}{\partial \eta}\left[g_{V}(\chi, \eta, \phi, T) \frac{\partial \tilde{V}_{001}(\chi, \eta, \phi, \vartheta)}{\partial \eta}\right]+\right.$

$\left.+\frac{\partial}{\partial \phi}\left[g_{V}(\chi, \eta, \phi, T) \frac{\partial \tilde{V}_{001}(\chi, \eta, \phi, \vartheta)}{\partial \phi}\right]\right\}-\left[1+\varepsilon_{V} g_{V}(\chi, \eta, \phi, T)\right] \tilde{I}_{000}(\chi, \eta, \phi, \vartheta) \tilde{V}_{100}(\chi, \eta, \phi, \vartheta)$

$\frac{\partial \tilde{I}_{011}(\chi, \eta, \phi, \vartheta)}{\partial \vartheta}=\sqrt{\frac{D_{01}}{D_{0 V}}}\left[\frac{\partial^{2} \tilde{I}_{011}(\chi, \eta, \phi, \vartheta)}{\partial \chi^{2}}+\frac{\partial^{2} \tilde{I}_{011}(\chi, \eta, \phi, \vartheta)}{\partial \eta^{2}}+\frac{\partial^{2} \tilde{I}_{011}(\chi, \eta, \phi, \vartheta)}{\partial \phi^{2}}\right]-\tilde{I}_{010}(\chi, \eta, \phi, \vartheta) \tilde{I}_{000}(\chi, \eta, \phi, \vartheta) \times$

$\times\left[1+\varepsilon_{I, I} g_{I, I}(\chi, \eta, \phi, T)\right]-\left[1+\varepsilon_{I, V} g_{I, V}(\chi, \eta, \phi, T)\right] \tilde{I}_{001}(\chi, \eta, \phi, \vartheta) \tilde{V}_{000}(\chi, \eta, \phi, \vartheta)$

$\frac{\partial \tilde{V}_{011}(\chi, \eta, \phi, \vartheta)}{\partial \vartheta}=\sqrt{\frac{D_{0 V}}{D_{01}}}\left[\frac{\partial^{2} \tilde{V}_{011}(\chi, \eta, \phi, \vartheta)}{\partial \chi^{2}}+\frac{\partial^{2} \tilde{V}_{011}(\chi, \eta, \phi, \vartheta)}{\partial \eta^{2}}+\frac{\partial^{2} \tilde{V}_{011}(\chi, \eta, \phi, \vartheta)}{\partial \phi^{2}}\right]-\tilde{V}_{010}(\chi, \eta, \phi, \vartheta) \tilde{V}_{000}(\chi, \eta, \phi, \vartheta) \times$

$\times\left[1+\varepsilon_{V, V} g_{V, V}(\chi, \eta, \phi, T)\right]-\left[1+\varepsilon_{I, V} g_{I, V}(\chi, \eta, \phi, t)\right] \tilde{I}_{000}(\chi, \eta, \phi, \vartheta) \tilde{V}_{001}(\chi, \eta, \phi, \vartheta)$

Conditions for considered functions are

$\left.\frac{\partial \tilde{\rho}_{i j k}(\chi, \eta, \phi, \vartheta)}{\partial \chi}\right|_{x=0}=0,\left.\frac{\partial \tilde{\rho}_{i j k}(\chi, \eta, \phi, \vartheta)}{\partial \chi}\right|_{x=1}=0,\left.\frac{\partial \tilde{\rho}_{i j k}(\chi, \eta, \phi, \vartheta)}{\partial \eta}\right|_{\eta=0}=0,\left.\frac{\partial \tilde{\rho}_{i j k}(\chi, \eta, \phi, \vartheta)}{\partial \eta}\right|_{\eta=1}=0$,

$\left.\frac{\partial \tilde{\rho}_{i j k}(\chi, \eta, \phi, \vartheta)}{\partial \phi}\right|_{\phi=0}=0,\left.\frac{\partial \tilde{\rho}_{i j k}(\chi, \eta, \phi, \vartheta)}{\partial \phi}\right|_{\phi=1}=0(i \geq 0, j \geq 0, k \geq 0)$

$\tilde{\rho}_{000}(\chi, \eta, \phi, 0)=f_{\rho}(\chi, \eta, \phi) / \rho^{*}, \tilde{\rho}_{i j k}(\chi, \eta, \phi, 0)=0 \quad(\mathrm{i} \geq 1, \mathrm{j} \geq 1, \mathrm{k} \geq 1)$

Solutions of the considered equations are

$\tilde{\rho}_{000}(\chi, \eta, \phi, \vartheta)=\frac{F_{0 \rho}}{L}+\frac{2}{L} \sum_{n=1}^{\infty} F_{n \rho} c(\chi) c(\eta) c(\phi) e_{n \rho}(\vartheta)$

Here $F_{n \rho}=\frac{1}{\rho^{*}} \int_{0}^{1} \cos (\pi n u) \int_{0}^{1} \cos (\pi n v) \int_{0}^{1} \cos (\pi n w) f_{n \rho}(u, v, w) d w d v d u, e_{n l}(\vartheta)=\exp \left(-\pi^{2} n^{2} \vartheta \sqrt{D_{0 V} / D_{0 I}}\right), c_{n}(\chi)$ $=\cos (\pi n \chi), e_{n V}(\vartheta)=\exp \left(-\pi^{2} n^{2} \vartheta \sqrt{D_{0 I} / D_{0 V}}\right) ;$

$\tilde{I}_{i 00}(\chi, \eta, \phi, \vartheta)=-2 \pi \sqrt{\frac{D_{0 I}}{D_{0 V}}} \sum_{n=1}^{\infty} n c_{n}(\chi) c(\eta) c(\phi) e_{n I}(\vartheta) \int_{0}^{\vartheta} e_{n I}(-\tau) \int_{0}^{1} s_{n}(u) \int_{0}^{1} c_{n}(v) \int_{0}^{1} \frac{\partial \tilde{I}_{i-100}(u, v, w, \tau)}{\partial u} \times$

$\times c_{n}(w) g_{I}(u, v, w, T) d w d v d u d \tau-2 \pi \sqrt{\frac{D_{0 I}}{D_{0 V}}} \sum_{n=1}^{\infty} n c_{n}(\chi) c(\eta) c(\phi) e_{n I}(\vartheta) \int_{0}^{\vartheta} e_{n I}(-\tau) \int_{0}^{1} c_{n}(u) \int_{0}^{1} s_{n}(v) \times$ 
$\times \int_{0}^{1} c_{n}(w) g_{I}(u, v, w, T) \frac{\partial \tilde{I}_{i-100}(u, v, w, \tau)}{\partial v} d w d v d u d \tau-2 \pi \sqrt{\frac{D_{0 I}}{D_{0 V}}} \sum_{n=1}^{\infty} n c_{n}(\chi) c(\eta) c(\phi) e_{n I}(\vartheta) \int_{0}^{g} e_{n I}(-\tau) \times$

$\times \int_{0}^{1} c_{n}(u) \int_{0}^{1} c_{n}(v) \int_{0}^{1} s_{n}(w) g_{I}(u, v, w, T) \frac{\partial \tilde{I}_{i-100}(u, v, w, \tau)}{\partial w} d w d v d u d \tau, i \geq 1$,

$\tilde{V}_{i 00}(\chi, \eta, \phi, \vartheta)=-2 \pi \sqrt{\frac{D_{0 V}}{D_{0 I}}} \sum_{n=1}^{\infty} n c_{n}(\chi) c(\eta) c(\phi) e_{n V}(\vartheta) \int_{0}^{\vartheta} e_{n I}(-\tau) \int_{0}^{1} s_{n}(u) \int_{0}^{1} c_{n}(v) \int_{0}^{1} g_{V}(u, v, w, T) \times$

$\times c_{n}(w) \frac{\partial \tilde{V}_{i-100}(u, \tau)}{\partial u} d w d v d u d \tau-\sqrt{\frac{D_{0 V}}{D_{0 I}}} \sum_{n=1}^{\infty} n c_{n}(\chi) c(\eta) c(\phi) e_{n V}(\vartheta) \int_{0}^{\vartheta} e_{n I}(-\tau) \int_{0}^{1} c_{n}(u) \int_{0}^{1} s_{n}(v) \times$

$\times \int_{0}^{\vartheta} e_{n I}(-\tau) \int_{0}^{1} c_{n}(u) \int_{0}^{1} c_{n}(v) \int_{0}^{1} s_{n}(w) g_{V}(u, v, w, T) \frac{\partial \tilde{V}_{i-100}(u, \tau)}{\partial w} d w d v d u d \tau, i \geq 1$

Here $s_{n}(\chi)$ is $\sin (\pi n \chi)$;

$\tilde{\rho}_{010}(\chi, \eta, \phi, \vartheta)=-2 \sum_{n=1}^{\infty} c_{n}(\chi) c_{n}(\eta) c_{n}(\phi) e_{n \rho}(\vartheta) \int_{0}^{\vartheta} e_{n \rho}(-\tau) \int_{0}^{1} c_{n}(u) \int_{0}^{1} c_{n}(v) \int_{0}^{1} c_{n}(w)\left[1+\varepsilon_{I, V} g_{I, V}(u, v, w, T)\right] \times$ $\times \tilde{I}_{000}(u, v, w, \tau) \tilde{V}_{000}(u, v, w, \tau) d w d v d u d \tau$

$\tilde{\rho}_{020}(\chi, \eta, \phi, \vartheta)=-2 \sqrt{\frac{D_{0 I}}{D_{0 V}}} \sum_{n=1}^{\infty} c_{n}(\chi) c_{n}(\eta) c_{n}(\phi) e_{n \rho}(\vartheta) \int_{0}^{\vartheta} e_{n \rho}(-\tau) \int_{0}^{1} c_{n}(u) \int_{0}^{1} c_{n}(v) \int_{0}^{1} c_{n}(w)\left[1+\varepsilon_{I, V} g_{I, V}(u, v, w, T)\right] \times$

$\times\left[\tilde{I}_{010}(u, v, w, \tau) \tilde{V}_{000}(u, v, w, \tau)+\tilde{I}_{000}(u, v, w, \tau) \tilde{V}_{010}(u, v, w, \tau)\right] d w d v d u d \tau$

$\tilde{\rho}_{001}(\chi, \eta, \phi, \vartheta)=-2 \sum_{n=1}^{\infty} c_{n}(\chi) c_{n}(\eta) c_{n}(\phi) e_{n \rho}(\vartheta) \int_{0}^{\vartheta} e_{n \rho}(-\tau) \int_{0}^{1} c_{n}(u) \int_{0}^{1} c_{n}(v) \int_{0}^{1} c_{n}(w) \times$

$\times\left[1+\varepsilon_{\rho, \rho} g_{\rho, \rho}(u, v, w, T)\right] \tilde{\rho}_{000}^{2}(u, v, w, \tau) d w d v d u d \tau$

$\tilde{\rho}_{002}(\chi, \eta, \phi, \vartheta)=-2 \sum_{n=1}^{\infty} c_{n}(\chi) c_{n}(\eta) c_{n}(\phi) e_{n \rho}(\vartheta) \int_{0}^{\vartheta} e_{n \rho}(-\tau) \int_{0}^{1} c_{n}(u) \int_{0}^{1} c_{n}(v) \int_{0}^{1} c_{n}(w) \times$

$\times\left[1+\varepsilon_{\rho, \rho} g_{\rho, \rho}(u, v, w, T)\right] \tilde{\rho}_{001}(u, v, w, \tau) \tilde{\rho}_{000}(u, v, w, \tau) d w d v d u d \tau$

$\tilde{I}_{110}(\chi, \eta, \phi, \vartheta)=-2 \pi \sqrt{\frac{D_{0 I}}{D_{0 V}}} \sum_{n=1}^{\infty} n c_{n}(\chi) c_{n}(\eta) c_{n}(\phi) e_{n I}(\vartheta) \int_{0}^{\vartheta} e_{n I}(-\tau) \int_{0}^{1} s_{n}(u) \int_{0}^{1} c_{n}(v) \int_{0}^{1} c_{n}(u) \times$

$\times g_{I}(u, v, w, T) \frac{\partial \tilde{I}_{i-100}(u, v, w, \tau)}{\partial u} d w d v d u d \tau-2 \pi \sqrt{\frac{D_{0 I}}{D_{0 V}}} \sum_{n=1}^{\infty} n c_{n}(\chi) c_{n}(\eta) c_{n}(\phi) e_{n I}(\vartheta) \times$ 
$\times \int_{0}^{g} e_{n I}(-\tau) \int_{0}^{1} c_{n}(u) \int_{0}^{1} s_{n}(v) \int_{0}^{1} c_{n}(u) g_{I}(u, v, w, T) \frac{\partial \tilde{I}_{i-100}(u, v, w, \tau)}{\partial v} d w d v d u d \tau-2 \pi \sqrt{\frac{D_{0 I}}{D_{0 V}}} \times$

$\times \sum_{n=1}^{\infty} n e_{n I}(\vartheta) \int_{0}^{\vartheta} e_{n I}(-\tau) \int_{0}^{1} c_{n}(u) \int_{0}^{1} c_{n}(v) \int_{0}^{1} s_{n}(u) g_{I}(u, v, w, T) \frac{\partial \tilde{I}_{i-100}(u, v, w, \tau)}{\partial w} d w d v d u d \tau \times$

$\times c_{n}(\chi) c_{n}(\eta) c_{n}(\phi)-2 \sum_{n=1}^{\infty} c_{n}(\chi) e_{n I}(\vartheta) c_{n}(\eta) c_{n}(\phi) \int_{0}^{g} e_{n I}(-\tau) \int_{0}^{1} c_{n}(u) \int_{0}^{1} c_{n}(v) \int_{0}^{1} c_{n}(v)\left[1+\varepsilon_{I, V} g_{I, V}(u, v, w, T)\right] \times$

$\left.\times g_{I, V}(u, v, w, T)\right]\left[\tilde{I}_{100}(u, v, w, \tau) \tilde{V}_{000}(u, v, w, \tau)+\tilde{I}_{000}(u, v, w, \tau) \tilde{V}_{100}(u, v, w, \tau)\right] d w d v d u d \tau$

$\tilde{V}_{110}(\chi, \eta, \phi, \vartheta)=-2 \pi \sqrt{\frac{D_{0 V}}{D_{0 I}}} \sum_{n=1}^{\infty} n c_{n}(\chi) c_{n}(\eta) c_{n}(\phi) e_{n V}(\vartheta) \int_{0}^{\vartheta} e_{n V}(-\tau) \int_{0}^{1} s_{n}(u) \int_{0}^{1} c_{n}(v) \int_{0}^{1} c_{n}(u) \times$

$\times g_{V}(u, v, w, T) \frac{\partial \tilde{V}_{i-100}(u, v, w, \tau)}{\partial u} d w d v d u d \tau-2 \pi \sqrt{\frac{D_{0 V}}{D_{0 I}}} \sum_{n=1}^{\infty} n c_{n}(\chi) c_{n}(\eta) c_{n}(\phi) e_{n V}(\vartheta) \times$

$\times \int_{0}^{g} e_{n V}(-\tau) \int_{0}^{1} c_{n}(u) \int_{0}^{1} s_{n}(v) \int_{0}^{1} c_{n}(u) g_{V}(u, v, w, T) \frac{\partial \tilde{V}_{i-100}(u, v, w, \tau)}{\partial v} d w d v d u d \tau-2 \pi \sqrt{\frac{D_{0 V}}{D_{0 I}}} \times$

$\times \sum_{n=1}^{\infty} n e_{n V}(\vartheta) \int_{0}^{\vartheta} e_{n V}(-\tau) \int_{0}^{1} c_{n}(u) \int_{0}^{1} c_{n}(v) \int_{0}^{1} s_{n}(u) g_{V}(u, v, w, T) \frac{\partial \tilde{V}_{i-100}(u, v, w, \tau)}{\partial w} d w d v d u d \tau \times$

$\times c_{n}(\chi) c_{n}(\eta) c_{n}(\phi)-2 \sum_{n=1}^{\infty} c_{n}(\chi) e_{n I}(\vartheta) c_{n}(\eta) c_{n}(\phi) \int_{0}^{\vartheta} e_{n V}(-\tau) \int_{0}^{1} c_{n}(u) \int_{0}^{1} c_{n}(v) \int_{0}^{1}\left[1+\varepsilon_{I, V} g_{I, V}(u, v, w, T)\right] \times$

$\times c_{n}(w)\left[\tilde{I}_{100}(u, v, w, \tau) \tilde{V}_{000}(u, v, w, \tau)+\tilde{I}_{000}(u, v, w, \tau) \tilde{V}_{100}(u, v, w, \tau)\right] d w d v d u d \tau$

$\tilde{I}_{101}(\chi, \eta, \phi, \vartheta)=-2 \pi \sqrt{\frac{D_{0 I}}{D_{0 V}}} \sum_{n=1}^{\infty} n c_{n}(\chi) c_{n}(\eta) c_{n}(\phi) e_{n I}(\vartheta) \int_{0}^{\vartheta} e_{n I}(-\tau) \int_{0}^{1} s_{n}(u) \int_{0}^{1} c_{n}(v) \int_{0}^{1} g_{I}(u, v, w, T) \times$

$\times c_{n}(w) \frac{\partial \tilde{I}_{001}(u, v, w, \tau)}{\partial u} d w d v d u d \tau-2 \pi \sqrt{\frac{D_{0 I}}{D_{0 V}}} \sum_{n=1}^{\infty} n c_{n}(\chi) c_{n}(\eta) c_{n}(\phi) e_{n I}(\vartheta) \times$

$\times \int_{0}^{1} s_{n}(v) \int_{0}^{1} c_{n}(w) g_{I}(u, v, w, T) \frac{\partial \tilde{I}_{001}(u, v, w, \tau)}{\partial v} d w d v d u d \tau-2 \pi \sqrt{\frac{D_{0 I}}{D_{0 V}}} \sum_{n=1}^{\infty} n e_{n I}(\vartheta) c_{n}(\chi) c_{n}(\eta) c_{n}(\phi) \times$

$\times \int_{0}^{\vartheta} e_{n I}(-\tau) \int_{0}^{1} c_{n}(u) \int_{0}^{1} c_{n}(v) \int_{0}^{1} s_{n}(w) g_{I}(u, v, w, T) \frac{\partial \tilde{I}_{001}(u, v, w, \tau)}{\partial w} d w d v d u d \tau-2 \sum_{n=1}^{\infty} c_{n}(\chi) c_{n}(\eta) c_{n}(\phi) \times$

$\times e_{n I}(\vartheta) \int_{0}^{\vartheta} e_{n I}(-\tau) \int_{0}^{1} c_{n}(u) \int_{0}^{1} c_{n}(v) \int_{0}^{1} c_{n}(w)\left[1+\varepsilon_{I, V} g_{I, V}(u, v, w, T)\right] \tilde{I}_{100}(u, v, w, \tau) \tilde{V}_{000}(u, v, w, \tau) d w d v d u d \tau$

$\tilde{V}_{101}(\chi, \eta, \phi, \vartheta)=-2 \pi \sqrt{\frac{D_{0 V}}{D_{0 I}}} \sum_{n=1}^{\infty} n c_{n}(\chi) c_{n}(\eta) c_{n}(\phi) e_{n V}(\vartheta) \int_{0}^{\vartheta} e_{n V}(-\tau) \int_{0}^{1} s_{n}(u) \int_{0}^{1} c_{n}(v) \int_{0}^{1} g_{V}(u, v, w, T) \times$ 
$\times c_{n}(w) \frac{\partial \tilde{V}_{001}(u, v, w, \tau)}{\partial u} d w d v d u d \tau-2 \pi \sqrt{\frac{D_{0 V}}{D_{0 I}}} \sum_{n=1}^{\infty} n c_{n}(\chi) c_{n}(\eta) c_{n}(\phi) e_{n I}(\vartheta) \int_{0}^{\vartheta} e_{n V}(-\tau) \int_{0}^{1} c_{n}(u) \times$ $\times \int_{0}^{1} s_{n}(v) \int_{0}^{1} c_{n}(w) g_{I}(u, v, w, T) \frac{\partial \tilde{I}_{001}(u, v, w, \tau)}{\partial v} d w d v d u d \tau-2 \pi \sqrt{\frac{D_{0 I}}{D_{0 V}}} \sum_{n=1}^{\infty} n e_{n I}(\vartheta) c_{n}(\chi) c_{n}(\eta) c_{n}(\phi) \times$ $\times \int_{0}^{g} e_{n V}(-\tau) \int_{0}^{1} c_{n}(u) \int_{0}^{1} c_{n}(v) \int_{0}^{1} s_{n}(w) g_{V}(u, v, w, T) \frac{\partial \tilde{V}_{001}(u, v, w, \tau)}{\partial w} d w d v d u d \tau-2 \sum_{n=1}^{\infty} c_{n}(\chi) c_{n}(\eta) c_{n}(\phi) \times$ $\times e_{n V}(\vartheta) \int_{0}^{\vartheta} e_{n V}(-\tau) \int_{0}^{1} c_{n}(u) \int_{0}^{1} c_{n}(v) \int_{0}^{1} c_{n}(w)\left[1+\varepsilon_{I, V} g_{I, V}(u, v, w, T)\right] \tilde{I}_{100}(u, v, w, \tau) \tilde{V}_{000}(u, v, w, \tau) d w d v d u d \tau$ $\tilde{I}_{011}(\chi, \eta, \phi, \vartheta)=-2 \sum_{n=1}^{\infty} c_{n}(\chi) c_{n}(\eta) c_{n}(\phi) e_{n I}(\vartheta) \int_{0}^{\vartheta} e_{n I}(-\tau) \int_{0}^{1} c_{n}(u) \int_{0}^{1} c_{n}(v) \int_{0}^{1} c_{n}(w)\left\{\tilde{I}_{000}(u, v, w, \tau) \times\right.$ $\left.\times\left[1+\varepsilon_{I, I} g_{I, I}(u, v, w, T)\right] \tilde{I}_{010}(u, v, w, \tau)+\left[1+\varepsilon_{I, V} g_{I, V}(u, v, w, T)\right] \tilde{I}_{001}(u, v, w, \tau) \tilde{V}_{000}(u, v, w, \tau)\right\} d w d v d u d \tau$ $\tilde{V}_{011}(\chi, \eta, \phi, \vartheta)=-2 \sum_{n=1}^{\infty} c_{n}(\chi) c_{n}(\eta) c_{n}(\phi) e_{n V}(\vartheta) \int_{0}^{\vartheta} e_{n V}(-\tau) \int_{0}^{1} c_{n}(u) \int_{0}^{1} c_{n}(v) \int_{0}^{1} c_{n}(w)\left\{\tilde{I}_{000}(u, v, w, \tau) \times\right.$ $\left.\times\left[1+\varepsilon_{I, I} g_{I, I}(u, v, w, T)\right] \tilde{I}_{010}(u, v, w, \tau)+\left[1+\varepsilon_{I, V} g_{I, V}(u, v, w, T)\right] \tilde{I}_{001}(u, v, w, \tau) \tilde{V}_{000}(u, v, w, \tau)\right\} d w d v d u d \tau$ Equations for the considered functions $\Phi_{\rho_{i}}(x, y, z, t), i \geq 0$ could be written as

$$
\begin{aligned}
& \frac{\partial \Phi_{I 0}(x, y, z, t)}{\partial t}=D_{0 \Phi I}\left[\frac{\partial^{2} \Phi_{I 0}(x, y, z, t)}{\partial x^{2}}+\frac{\partial^{2} \Phi_{I 0}(x, y, z, t)}{\partial y^{2}}+\frac{\partial^{2} \Phi_{I 0}(x, y, z, t)}{\partial z^{2}}\right]+ \\
& +k_{I, I}(x, y, z, T) I^{2}(x, y, z, t)-k_{I}(x, y, z, T) I(x, y, z, t) \\
& \frac{\partial \Phi_{V 0}(x, y, z, t)}{\partial t}=D_{0 \Phi V}\left[\frac{\partial^{2} \Phi_{V 0}(x, y, z, t)}{\partial x^{2}}+\frac{\partial^{2} \Phi_{V 0}(x, y, z, t)}{\partial y^{2}}+\frac{\partial^{2} \Phi_{V 0}(x, y, z, t)}{\partial z^{2}}\right]+ \\
& +k_{V, V}(x, y, z, T) V^{2}(x, y, z, t)-k_{V}(x, y, z, T) V(x, y, z, t) \\
& \frac{\partial \Phi_{I i}(x, y, z, t)}{\partial t}=D_{0 \Phi I}\left[\frac{\partial^{2} \Phi_{I i}(x, y, z, t)}{\partial x^{2}}+\frac{\partial^{2} \Phi_{I i}(x, y, z, t)}{\partial y^{2}}+\frac{\partial^{2} \Phi_{I i}(x, y, z, t)}{\partial z^{2}}\right]+D_{0 \Phi I}\left\{\frac { \partial } { \partial x } \left[g_{\Phi I}(x, y, z, T) \times\right.\right. \\
& \left.\left.\times \frac{\partial \Phi_{I i-1}(x, y, z, t)}{\partial y}\right]+\frac{\partial}{\partial y}\left[g_{\Phi I}(x, y, z, T) \frac{\partial \Phi_{I i-1}(x, y, z, t)}{\partial y}\right]+\frac{\partial}{\partial z}\left[g_{\Phi I}(x, y, z, T) \frac{\partial \Phi_{I i-1}(x, y, z, t)}{\partial z}\right]\right\}, i \geq 1, \\
& \frac{\partial \Phi_{V i}(x, y, z, t)}{\partial t}=D_{0 \Phi V}\left[\frac{\partial^{2} \Phi_{V i}(x, y, z, t)}{\partial x^{2}}+\frac{\partial^{2} \Phi_{V i}(x, y, z, t)}{\partial y^{2}}+\frac{\partial^{2} \Phi_{V i}(x, y, z, t)}{\partial z^{2}}\right]+D_{0 \Phi V}\left\{\frac { \partial } { \partial x } \left[g_{\Phi V}(x, y, z, T) \times\right.\right.
\end{aligned}
$$




$$
\left.\left.\times \frac{\partial \Phi_{V i-1}(x, y, z, t)}{\partial y}\right]+\frac{\partial}{\partial y}\left[g_{\Phi V}(x, y, z, T) \frac{\partial \Phi_{V i-1}(x, y, z, t)}{\partial y}\right]+\frac{\partial}{\partial z}\left[g_{\Phi V}(x, y, z, T) \frac{\partial \Phi_{V i-1}(x, y, z, t)}{\partial z}\right]\right\}, i \geq 1,
$$

Initial and boundary conditions for the considered functions could be written as

$$
\begin{aligned}
& \left.\frac{\partial \Phi_{\rho i}(x, y, z, t)}{\partial x}\right|_{x=0}=0,\left.\frac{\partial \Phi_{\rho i}(x, y, z, t)}{\partial x}\right|_{x=L_{x}}=0,\left.\frac{\partial \Phi_{\rho i}(x, y, z, t)}{\partial y}\right|_{y=0}=0,\left.\frac{\partial \Phi_{\rho i}(x, y, z, t)}{\partial y}\right|_{y=L_{y}}=0, \\
& \left.\frac{\partial \Phi_{\rho i}(x, y, z, t)}{\partial z}\right|_{z=0}=0,\left.\frac{\partial \Phi_{\rho i}(x, y, z, t)}{\partial z}\right|_{z=L_{z}}=0, \mathrm{i} \geq 0 ; \Phi_{\rho o}(\mathrm{x}, \mathrm{y}, \mathrm{z}, 0)=\mathrm{f}_{\Phi_{\rho}}(\mathrm{x}, \mathrm{y}, \mathrm{z}), \Phi_{\rho \mathrm{i}}(\mathrm{x}, \mathrm{y}, \mathrm{z}, 0)=0, \mathrm{i} \geq 1 .,
\end{aligned}
$$

Solutions of the considered equations are

$$
\begin{aligned}
& \Phi_{\rho 0}(x, y, z, t)=\frac{F_{0 \Phi_{\rho}}}{L_{x} L_{y} L_{z}}+\frac{2}{L_{x} L_{y} L_{z}} \sum_{n=1}^{\infty} F_{n \Phi_{\rho}} c_{n}(x) c_{n}(y) c_{n}(z) e_{n \Phi_{\rho}}(t)+\frac{2}{L} \sum_{n=1}^{\infty} n c_{n}(x) c_{n}(y) c_{n}(z) e_{\Phi_{\rho} n}(t) \times \\
& \times \int_{0}^{t} e_{\Phi_{\rho} n}(-\tau) \int_{0}^{L_{x}} c_{n}(u) \int_{0}^{L_{y}} c_{n}(v) \int_{0}^{L_{z}} c_{n}(w)\left[k_{I, I}(u, v, w, T) I^{2}(u, v, w, \tau)-k_{I}(u, v, w, T) I(u, v, w, \tau)\right] d w d v d u d \tau
\end{aligned}
$$

Here $\left.F_{n \Phi_{\rho}}=\int_{0}^{L_{x}} c_{n}(u) \int_{0}^{L_{y}} c_{n}(v) \int_{0}^{L_{z}} c_{n}(w) f_{\Phi_{\rho}}(u, v, w) d w d v d u, e_{n \Phi_{\rho}}(t)=\exp L-\pi^{2} n^{2} D_{0 \Phi_{\rho}} t\left(L_{x}^{-2}+L_{y}^{-2}+L_{z}^{-2}\right)\right]$

$$
\begin{aligned}
& \Phi_{\rho i}(x, y, z, t)=-\frac{2 \pi}{L_{x}^{2} L_{y} L_{z}} \sum_{n=1}^{\infty} n c_{n}(x) c_{n}(y) c_{n}(z) e_{\Phi_{\rho} n}(t) \int_{0}^{t} e_{\Phi_{\rho} n}(-\tau) \int_{0}^{L_{x}} s_{n}(u) \int_{0}^{L_{y}} c_{n}(v) \int_{0}^{L_{z}} g_{\Phi_{\rho}}(u, v, w, T) \times \\
& \times c_{n}(w) \frac{\partial \Phi_{I_{\rho} i-1}(u, v, w, \tau)}{\partial u} d w d v d u d \tau-\frac{2 \pi}{L_{x} L_{y}^{2} L_{z}} \sum_{n=1}^{\infty} n c_{n}(x) c_{n}(y) c_{n}(z) e_{\Phi_{\rho^{n}}}(t) \int_{0}^{t} e_{\Phi_{\rho^{n}}}(-\tau) \times \\
& \times \int_{0}^{t} e_{\Phi_{\rho^{n}}}(-\tau) \int_{0}^{L_{x}} c_{n}(u) \int_{0}^{L_{y}} s_{n}(v) \int_{0}^{L_{z}} c_{n}(w) g_{\Phi_{\rho}}(u, v, w, T) \frac{\partial \Phi_{\rho_{\rho} i-1}(u, v, w, \tau)}{\partial v} d w d v d u d \tau-\frac{2 \pi}{L_{x} L_{y} L_{z}^{2}} \sum_{n=1}^{\infty} n c_{n}(x) c_{n}(y) \times \\
& \times c_{n}(z) e_{\Phi_{\rho^{n}}}(t) \int_{0}^{t} e_{\Phi_{\rho^{n}}}(-\tau) \int_{0}^{L_{x}} c_{n}(u) \int_{0}^{L_{y}} c_{n}(v) \int_{0}^{L_{z}} s_{n}(w) \frac{\partial \Phi_{I_{\rho} i-1}(u, v, w, \tau)}{\partial w} g_{\Phi_{\rho}}(u, v, w, T) d w d v d u d \tau, i \geq 1 .
\end{aligned}
$$

Functions $C_{i j}(x, y, z, t)$ describes by the following equations for any values of indexes $i \geq 0, j \geq 0$

$$
\begin{aligned}
& \frac{\partial C_{00}(x, y, z, t)}{\partial t}=D_{0 L} \frac{\partial^{2} C_{00}(x, y, z, t)}{\partial x^{2}}+D_{0 L} \frac{\partial^{2} C_{00}(x, y, z, t)}{\partial y^{2}}+D_{0 L} \frac{\partial^{2} C_{00}(x, y, z, t)}{\partial z^{2}} \\
& \frac{\partial C_{i 0}(x, y, z, t)}{\partial t}=D_{0 L}\left[\frac{\partial^{2} C_{i 0}(x, y, z, t)}{\partial x^{2}}+\frac{\partial^{2} C_{i 0}(x, y, z, t)}{\partial y^{2}}+\frac{\partial^{2} C_{i 0}(x, y, z, t)}{\partial z^{2}}\right]+D_{0 L} \frac{\partial}{\partial x}\left[g_{L}(x, y, z, T) \times\right. \\
& \frac{\partial C_{01}(x, y, z, t)}{\partial t}=D_{0 L} \frac{\partial^{2} C_{01}(x, y, z, t)}{\partial x^{2}}+D_{0 L} \frac{\partial^{2} C_{01}(x, y, z, t)}{\partial y^{2}}+D_{0 L} \frac{\partial^{2} C_{01}(x, y, z, t)}{\partial z^{2}}+
\end{aligned}
$$


$+D_{0 L} \frac{\partial}{\partial x}\left[\frac{C_{00}^{\gamma}(x, y, z, t)}{P^{\gamma}(x, y, z, T)} \frac{\partial C_{00}(x, y, z, t)}{\partial x}\right]+D_{0 L} \frac{\partial}{\partial y}\left[\frac{C_{00}^{\gamma}(x, y, z, t)}{P^{\gamma}(x, y, z, T)} \frac{\partial C_{00}(x, y, z, t)}{\partial y}\right]+$

$+D_{0 L} \frac{\partial}{\partial z}\left[\frac{C_{00}^{\gamma}(x, y, z, t)}{P^{\gamma}(x, y, z, T)} \frac{\partial C_{00}(x, y, z, t)}{\partial z}\right]$

$\frac{\partial C_{02}(x, y, z, t)}{\partial t}=D_{0 L} \frac{\partial^{2} C_{02}(x, y, z, t)}{\partial x^{2}}+D_{0 L} \frac{\partial^{2} C_{02}(x, y, z, t)}{\partial y^{2}}+D_{0 L} \frac{\partial^{2} C_{02}(x, y, z, t)}{\partial z^{2}}+$

$+D_{0 L}\left\{\frac{\partial}{\partial x}\left[C_{01}(x, y, z, t) \frac{C_{00}^{\gamma-1}(x, y, z, t)}{P^{\gamma}(x, y, z, T)} \frac{\partial C_{00}(x, y, z, t)}{\partial x}\right]+\frac{\partial}{\partial y}\left[C_{01}(x, y, z, t) \frac{C_{00}^{\gamma-1}(x, y, z, t)}{P^{\gamma}(x, y, z, T)} \frac{\partial C_{00}(x, y, z, t)}{\partial y}\right]+\right.$

$\left.+\frac{\partial}{\partial z}\left[C_{01}(x, y, z, t) \frac{C_{00}^{\gamma-1}(x, y, z, t)}{P^{\gamma}(x, y, z, T)} \frac{\partial C_{00}(x, y, z, t)}{\partial z}\right]\right\}+D_{0 L}\left\{\frac{\partial}{\partial x}\left[\frac{C_{00}^{\gamma}(x, y, z, t)}{P^{\gamma}(x, y, z, T)} \frac{\partial C_{00}(x, y, z, t)}{\partial x}\right]+\right.$

$\left.+\frac{\partial}{\partial y}\left[\frac{C_{00}^{\gamma}(x, y, z, t)}{P^{\gamma}(x, y, z, T)} \frac{\partial C_{01}(x, y, z, t)}{\partial y}\right]+\frac{\partial}{\partial z}\left[\frac{C_{00}^{\gamma}(x, y, z, t)}{P^{\gamma}(x, y, z, T)} \frac{\partial C_{01}(x, y, z, t)}{\partial z}\right]\right\}$

$\frac{\partial C_{11}(x, y, z, t)}{\partial t}=D_{0 L} \frac{\partial^{2} C_{11}(x, y, z, t)}{\partial x^{2}}+D_{0 L} \frac{\partial^{2} C_{11}(x, y, z, t)}{\partial y^{2}}+D_{0 L} \frac{\partial^{2} C_{11}(x, y, z, t)}{\partial z^{2}}+$

$+D_{0 L}\left\{\frac{\partial}{\partial x}\left[C_{10}(x, y, z, t) \frac{C_{00}^{\gamma-1}(x, y, z, t)}{P^{\gamma}(x, y, z, T)} \frac{\partial C_{00}(x, y, z, t)}{\partial x}\right]+\frac{\partial}{\partial y}\left[C_{10}(x, y, z, t) \frac{C_{00}^{\gamma-1}(x, y, z, t)}{P^{\gamma}(x, y, z, T)} \frac{\partial C_{00}(x, y, z, t)}{\partial y}\right]+\right.$

$\left.+\frac{\partial}{\partial z}\left[C_{10}(x, y, z, t) \frac{C_{00}^{\gamma-1}(x, y, z, t)}{P^{\gamma}(x, y, z, T)} \frac{\partial C_{00}(x, y, z, t)}{\partial z}\right]\right\}+D_{0 L}\left\{\frac{\partial}{\partial x}\left[\frac{C_{00}^{\gamma}(x, y, z, t)}{P^{\gamma}(x, y, z, T)} \frac{\partial C_{10}(x, y, z, t)}{\partial x}\right]+\right.$

$\left.+\frac{\partial}{\partial y}\left[\frac{C_{00}^{y}(x, y, z, t)}{P^{\gamma}(x, y, z, T)} \frac{\partial C_{10}(x, y, z, t)}{\partial y}\right]+\frac{\partial}{\partial z}\left[\frac{C_{00}^{\gamma}(x, y, z, t)}{P^{\gamma}(x, y, z, T)} \frac{\partial C_{10}(x, y, z, t)}{\partial z}\right]\right\}+D_{0 L}\left\{\frac{\partial}{\partial x}\left[g_{L}(x, y, z, T) \frac{\partial C_{01}(x, y, z, t)}{\partial x}\right]+\right.$

$\left.+\frac{\partial}{\partial y}\left[g_{L}(x, y, z, T) \frac{\partial C_{01}(x, y, z, t)}{\partial y}\right]+\frac{\partial}{\partial z}\left[g_{L}(x, y, z, T) \frac{\partial C_{01}(x, y, z, t)}{\partial z}\right]\right\}$

Initial and boundary conditions for the considered functions takes the form

$$
\begin{gathered}
\left.\frac{\partial C_{i j}(x, y, z, t)}{\partial x}\right|_{x=0}=0,\left.\frac{\partial C_{i j}(x, y, z, t)}{\partial x}\right|_{x=L_{x}}=0,\left.\frac{\partial C_{i j}(x, y, z, t)}{\partial y}\right|_{y=0}=0,\left.\frac{\partial C_{i j}(x, y, z, t)}{\partial y}\right|_{y=L_{y}}=0, \\
\left.\frac{\partial C_{i j}(x, y, z, t)}{\partial z}\right|_{z=0}=0,\left.\frac{\partial C_{i j}(x, y, z, t)}{\partial z}\right|_{z=L_{z}}=0, i \geq 0, j \geq 0 ; C_{00}(x, y, z, 0)=f_{C}(x, y, z), C_{i j}(x, y, z, 0)=0, i \geq 1, j \geq 1 .
\end{gathered}
$$

Solutions for the above equations are 
$C_{00}(x, y, z, t)=\frac{F_{0 C}}{L_{x} L_{y} L_{z}}+\frac{2}{L_{x} L_{y} L_{z}} \sum_{n=1}^{\infty} F_{n C} c_{n}(x) c_{n}(y) c_{n}(z) e_{n C}(t)$

Here $e_{n C}(t)=\exp \left[-\pi^{2} n^{2} D_{0 c} t\left(\frac{1}{L_{x}^{2}}+\frac{1}{L_{y}^{2}}+\frac{1}{L_{z}^{2}}\right)\right], F_{n C}=\int_{0}^{L_{x}} c_{n}(u) \int_{0}^{L_{y}} c_{n}(v) \int_{0}^{L_{z}} f_{C}(u, v, w) c_{n}(w) d w d v d u$;

$C_{i 0}(x, y, z, t)=-\frac{2 \pi}{L_{x}^{2} L_{y} L_{z}} \sum_{n=1}^{\infty} n F_{n C} c_{n}(x) c_{n}(y) c_{n}(z) e_{n C}(t) \int_{0}^{t} e_{n C}(-\tau) \int_{0}^{L_{x}} s_{n}(u) \int_{0}^{L_{y}} c_{n}(v) \int_{0}^{L_{z}} g_{L}(u, v, w, T) \times$

$\times c_{n}(w) \frac{\partial C_{i-10}(u, v, w, \tau)}{\partial u} d w d v d u d \tau-\frac{2 \pi}{L_{x} L_{y}^{2} L_{z}} \sum_{n=1}^{\infty} n F_{n C} c_{n}(x) c_{n}(y) c_{n}(z) e_{n C}(t) \int_{0}^{t} e_{n C}(-\tau) \times$

$\times \int_{0}^{L_{x}} c_{n}(u) \int_{0}^{L_{y}} S_{n}(v) \int_{0}^{L_{z}} c_{n}(v) g_{L}(u, v, w, T) \frac{\partial C_{i-10}(u, v, w, \tau)}{\partial v} d w d v d u d \tau-\frac{2 \pi}{L_{x} L_{y} L_{z}^{2}} \sum_{n=1}^{\infty} n F_{n C} e_{n C}(t) \times$

$\times c_{n}(x) c_{n}(y) c_{n}(z) \int_{0}^{t} e_{n C}(-\tau) \int_{0}^{L_{x}} c_{n}(u) \int_{0}^{L_{y}} c_{n}(v) \int_{0}^{L_{z}} s_{n}(v) g_{L}(u, v, w, T) \frac{\partial C_{i-10}(u, v, w, \tau)}{\partial w} d w d v d u d \tau$

$C_{01}(x, y, z, t)=-\frac{2 \pi}{L_{x}^{2} L_{y} L_{z}} \sum_{n=1}^{\infty} n F_{n C} c_{n}(x) c_{n}(y) c_{n}(z) e_{n C}(t) \int_{0}^{t} e_{n C}(-\tau) \int_{0}^{L_{x}} s_{n}(u) \int_{0}^{L_{y}} c_{n}(v) \int_{0}^{L_{z}} c_{n}(w) \times$

$\times \frac{C_{00}^{\gamma}(u, v, w, \tau)}{P^{\gamma}(u, v, w, T)} \frac{\partial C_{00}(u, v, w, \tau)}{\partial u} d w d v d u d \tau-\frac{2 \pi}{L_{x} L_{y}^{2} L_{z}} \sum_{n=1}^{\infty} n F_{n C} c_{n}(x) c_{n}(y) c_{n}(z) e_{n C}(t) \times$

$\times \int_{0}^{t} e_{n C}(-\tau) \int_{0}^{L_{x}} c_{n}(u) \int_{0}^{L_{y}} s_{n}(v) \int_{0}^{L_{z}} c_{n}(w) \frac{C_{00}^{\gamma}(u, v, w, \tau)}{P^{\gamma}(u, v, w, T)} \frac{\partial C_{00}(u, v, w, \tau)}{\partial v} d w d v d u d \tau-\frac{2 \pi}{L_{x} L_{y} L_{z}^{2}} \sum_{n=1}^{\infty} n e_{n C}(t) \times$

$\times F_{n C} c_{n}(x) c_{n}(y) c_{n}(z) \int_{0}^{t} e_{n C}(-\tau) \int_{0}^{L_{x}} c_{n}(u) \int_{0}^{L_{y}} c_{n}(v) \int_{0}^{L_{z}} s_{n}(w) \frac{C_{00}^{\gamma}(u, v, w, \tau)}{P^{\gamma}(u, v, w, T)} \frac{\partial C_{00}(u, v, w, \tau)}{\partial w} d w d v d u d \tau$

$C_{02}(x, y, z, t)=-\frac{2 \pi}{L_{x}^{2} L_{y} L_{z}} \sum_{n=1}^{\infty} n F_{n C} c_{n}(x) c_{n}(y) c_{n}(z) e_{n C}(t) \int_{0}^{t} e_{n C}(-\tau) \int_{0}^{L_{x}} s_{n}(u) \int_{0}^{L_{y}} c_{n}(v) \int_{0}^{L_{z}} c_{n}(w) \times$

$\times C_{01}(u, v, w, \tau) \frac{C_{00}^{\gamma-1}(u, v, w, \tau)}{P^{\gamma}(u, v, w, T)} \frac{\partial C_{00}(u, v, w, \tau)}{\partial u} d w d v d u d \tau-\frac{2 \pi}{L_{x} L_{y}^{2} L_{z}} \sum_{n=1}^{\infty} F_{n C} c_{n}(x) c_{n}(y) \times$

$\times n c_{n}(z) e_{n C}(t) \int_{0}^{t} e_{n C}(-\tau) \int_{0}^{L_{x}} c_{n}(u) \int_{0}^{L_{y}} s_{n}(v) \int_{0}^{L_{z}} C_{01}(u, v, w, \tau) \frac{C_{00}^{\gamma-1}(u, v, w, \tau)}{P^{\gamma}(u, v, w, T)} \frac{\partial C_{00}(u, v, w, \tau)}{\partial v} \times$

$\times c_{n}(w) d w d v d u d \tau-\frac{2 \pi}{L_{x} L_{y} L_{z}^{2}} \sum_{n=1}^{\infty} n F_{n C} c_{n}(x) c_{n}(y) c_{n}(z) e_{n C}(t) \int_{0}^{t} e_{n C}(-\tau) \int_{0}^{L_{x}} c_{n}(u) \int_{0}^{L_{y}} c_{n}(v) \times$

$\times \int_{0}^{L_{z}} s_{n}(w) C_{01}(u, v, w, \tau) \frac{C_{00}^{\gamma-1}(u, v, w, \tau)}{P^{\gamma}(u, v, w, T)} \frac{\partial C_{00}(u, v, w, \tau)}{\partial w} d w d v d u d \tau-\frac{2 \pi}{L_{x}^{2} L_{y} L_{z}} \sum_{n=1}^{\infty} n c_{n}(x) \times$ 
$\times F_{n C} c_{n}(y) c_{n}(z) e_{n C}(t) \int_{0}^{t} e_{n C}(-\tau) \int_{0}^{L_{x}} s_{n}(u) \int_{0}^{L_{y}} c_{n}(v) \int_{0}^{L_{z}} c_{n}(w) C_{01}(u, v, w, \tau) \frac{\partial C_{00}(u, v, w, \tau)}{\partial u} \times$

$\times \frac{C_{00}^{\gamma-1}(u, v, w, \tau)}{P^{\gamma}(u, v, w, T)} d w d v d u d \tau-\frac{2 \pi}{L_{x} L_{y}^{2} L_{z}} \sum_{n=1}^{\infty} n F_{n C} c_{n}(x) c_{n}(y) c_{n}(z) e_{n C}(t) \int_{0}^{t} e_{n C}(-\tau) \int_{0}^{L_{x}} c_{n}(u) \times$

$\times \int_{0}^{L_{y}} S_{n}(v) \int_{0}^{L_{z}} c_{n}(w) C_{01}(u, v, w, \tau) \frac{C_{00}^{\gamma-1}(u, v, w, \tau)}{P^{\gamma}(u, v, w, T)} \frac{\partial C_{00}(u, v, w, \tau)}{\partial v} d w d v d u d \tau-\frac{2 \pi}{L_{x} L_{y} L_{z}^{2}} \sum_{n=1}^{\infty} n \times$

$\times F_{n C} c_{n}(x) c_{n}(y) c_{n}(z) e_{n C}(t) \int_{0}^{t} e_{n C}(-\tau) \int_{0}^{L_{x}} c_{n}(u) \int_{0}^{L_{y}} c_{n}(v) \int_{0}^{L_{z}} s_{n}(w) C_{01}(u, v, w, \tau) \frac{C_{00}^{\gamma-1}(u, v, w, \tau)}{P^{\gamma}(u, v, w, T)} \times$

$\times \frac{\partial C_{00}(u, v, w, \tau)}{\partial w} d w d v d u d \tau-\frac{2 \pi}{L_{x}^{2} L_{y} L_{z}} \sum_{n=1}^{\infty} n F_{n C} c_{n}(x) c_{n}(y) c_{n}(z) e_{n C}(t) \int_{0}^{t} e_{n C}(-\tau) \int_{0}^{L_{x}} s_{n}(u) \int_{0}^{L_{y}} c_{n}(v) \times$

$\times \int_{0}^{L_{y}} c_{n}(v) \int_{0}^{L_{z}} c_{n}(w) \frac{C_{00}^{\gamma}(u, v, w, \tau)}{P^{\gamma}(u, v, w, T)} \frac{\partial C_{01}(u, v, w, \tau)}{\partial u} d w d v d u d \tau-\frac{2 \pi}{L_{x} L_{y}^{2} L_{z}} \sum_{n=1}^{\infty} n F_{n C} c_{n}(x) c_{n}(y) c_{n}(z) e_{n C}(t) \times$

$\times \int_{0}^{t} e_{n C}(-\tau) \int_{0}^{L_{x}} c_{n}(u) \int_{0}^{L_{y}} s_{n}(v) \int_{0}^{L_{z}} c_{n}(w) \frac{C_{00}^{\gamma}(u, v, w, \tau)}{P^{\gamma}(u, v, w, T)} \frac{\partial C_{01}(u, v, w, \tau)}{\partial v} d w d v d u d \tau-\frac{2 \pi}{L_{x} L_{y} L_{z}^{2}} \sum_{n=1}^{\infty} n F_{n C} c_{n}(x) c_{n}(y) \times$

$\times c_{n}(z) e_{n C}(t) \int_{0}^{t} e_{n C}(-\tau) \int_{0}^{L_{x}} c_{n}(u) \int_{0}^{L_{y}} c_{n}(v) \int_{0}^{L_{z}} s_{n}(w) \frac{C_{00}^{\gamma}(u, v, w, \tau)}{P^{\gamma}(u, v, w, T)} \frac{\partial C_{01}(u, v, w, \tau)}{\partial w} d w d v d u d \tau$

$C_{11}(x, y, z, t)=-\frac{2 \pi}{L_{x}^{2} L_{y} L_{z}} \sum_{n=1}^{\infty} n F_{n C} c_{n}(x) c_{n}(y) c_{n}(z) e_{n C}(t) \int_{0}^{t} e_{n C}(-\tau) \int_{0}^{L_{x}} S_{n}(u) \int_{0}^{L_{y}} c_{n}(v) \int_{0}^{L_{z}} c_{n}(w) \frac{\partial C_{01}(u, v, w, \tau)}{\partial u} \times$

$\times g_{L}(u, v, w, T) d w d v d u d \tau-\frac{2 \pi}{L_{x} L_{y}^{2} L_{z}} \sum_{n=1}^{\infty} n F_{n C} c_{n}(x) c_{n}(y) c_{n}(z) e_{n C}(t) \int_{0}^{t} e_{n C}(-\tau) \int_{0}^{L_{x}} c_{n}(u) \int_{0}^{L_{y}} s_{n}(v) \int_{0}^{L_{z}} c_{n}(w) \times$

$\times g_{L}(u, v, w, T) \frac{\partial C_{01}(u, v, w, \tau)}{\partial v} d w d v d u d \tau-\frac{2 \pi}{L_{x} L_{y} L_{z}^{2}} \sum_{n=1}^{\infty} n F_{n C} c_{n}(x) c_{n}(y) c_{n}(z) e_{n C}(t) \int_{0}^{t} e_{n C}(-\tau) \int_{0}^{L_{x}} c_{n}(u) \int_{0}^{L_{y}} c_{n}(v) \times$

$\times \int_{0}^{L_{z}} s_{n}(w) g_{L}(u, v, w, T) \frac{\partial C_{01}(u, v, w, \tau)}{\partial w} d w d v d u d \tau-\frac{2 \pi}{L_{x}^{2} L_{v} L_{z}} \sum_{n=1}^{\infty} n F_{n C} c_{n}(x) c_{n}(y) c_{n}(z) e_{n C}(t) \int_{0}^{t} e_{n C}(-\tau) \int_{0}^{L_{x}} s_{n}(u) \times$

$\times n \int_{0}^{L_{y}} c_{n}(v) \int_{0}^{L_{z}} c_{n}(w) \frac{C_{00}^{\gamma}(u, v, w, \tau)}{P^{\gamma}(u, v, w, T)} \frac{\partial C_{10}(u, v, w, \tau)}{\partial u} d w d v d u d \tau-\frac{2 \pi}{L_{x} L_{y}^{2} L_{z}} \sum_{n=1}^{\infty} n c_{n}(x) c_{n}(y) c_{n}(z) e_{n C}(t) \int_{0}^{t} e_{n C}(-\tau) \times$

$\times F_{n C} \int_{0}^{L_{x}} c_{n}(u) \int_{0}^{L_{y}} s_{n}(v) \int_{0}^{L_{z}} c_{n}(w) \frac{C_{00}^{\gamma}(u, v, w, \tau)}{P^{\gamma}(u, v, w, T)} \frac{\partial C_{10}(u, v, w, \tau)}{\partial v} d w d v d u d \tau-\frac{2 \pi}{L_{x} L_{y} L_{z}^{2}} \sum_{n=1}^{\infty} F_{n C} c_{n}(x) c_{n}(y) c_{n}(z) e_{n C}(t) \times$

$\times n \int_{0}^{t} e_{n C}(-\tau) \int_{0}^{L_{x}} c_{n}(u) \int_{0}^{L_{y}} c_{n}(v) \int_{0}^{L_{z}} s_{n}(w) \frac{C_{00}^{\gamma}(u, v, w, \tau)}{P^{\gamma}(u, v, w, T)} \frac{\partial C_{10}(u, v, w, \tau)}{\partial w} d w d v d u d \tau-\frac{2 \pi}{L_{x}^{2} L_{y} L_{z}} \sum_{n=1}^{\infty} F_{n C} c_{n}(x) c_{n}(y) c_{n}(z) \times$ 


$$
\begin{aligned}
& \times n e_{n C}(t) \int_{0}^{t} e_{n C}(-\tau) \int_{0}^{L_{x}} s_{n}(u) \int_{0}^{L_{y}} c_{n}(v) \int_{0}^{L_{z}} c_{n}(w) C_{10}(u, v, w, \tau) \frac{C_{00}^{\gamma-1}(u, v, w, \tau)}{P^{\gamma}(u, v, w, T)} \frac{\partial C_{00}(u, v, w, \tau)}{\partial u} d w d v d u d \tau-\frac{2 \pi}{L_{x} L_{y}^{2} L_{z}} \times \\
& \times \sum_{n=1}^{\infty} n F_{n C} c_{n}(x) c_{n}(y) c_{n}(z) e_{n C}(t) \int_{0}^{t} e_{n C}(-\tau) \int_{0}^{L_{x}} c_{n}(u) \int_{0}^{L_{y}} s_{n}(v) \int_{0}^{L_{z}} C_{10}(u, v, w, \tau) \frac{C_{00}^{\gamma-1}(u, v, w, \tau)}{P^{\gamma}(u, v, w, T)} \frac{\partial C_{00}(u, v, w, \tau)}{\partial v} \times \\
& \times c_{n}(w) d w d v d u d \tau-\frac{2 \pi}{L_{x} L_{y} L_{z}^{2}} \sum_{n=1}^{\infty} n F_{n C} c_{n}(x) c_{n}(y) c_{n}(z) e_{n C}(t) \int_{0}^{t} e_{n C}(-\tau) \int_{0}^{L_{x}} c_{n}(u) \int_{0}^{L_{y}} c_{n}(v) \int_{0}^{L_{z}} s_{n}(w) C_{10}(u, v, w, \tau) \times \\
& \times \frac{C_{00}^{\gamma-1}(u, v, w, \tau)}{P^{\gamma}(u, v, w, T)} \frac{\partial C_{00}(u, v, w, \tau)}{\partial w} d w d v d u d \tau
\end{aligned}
$$

\section{REFERENCES}

[1] V. I. Lachin, N.S. Savelov. Electronics, Phoenix, Rostov-na-Donu, 2001.

[2] E. P. Ugryumov. Digital circuitry, Radio and communication, Moscow, BHV-Peterburg, 2010.

[3] V. A. Emel'anov, A.S. Turtsevich, A.I. Belous, Basis of circuitry for microelectronic devices, Radio and communication, Moscow, 2012.

[4] R. R. Ghiasi, A. Sahafi, J. S. Geshlaghi, Z. D. Kouzekanani, “A 2:1 switched-capacitor DC-DC converter for low power circuits," Analog Integrated Circuits and Signal Processing, vol. 84, pp. 215-222. 2015. [Online]. Available: https://link.springer.com/article/10.1007/s10470-015-0551-x.

[5] D. Fathi, B. Forouzandeh, N. Masoumi, "New enhanced noise analysis in active mixers in nanoscale technologies," Nano, vol. 4, no. 4, pp. 233-238. 2009. [Online]. Available: https://www.worldscientific.com/doi/abs/10.1142/S1793292009001708.

[6] S. A. Chachuli, P. N. A. Fasyar, N. Soin, N. M. Karim, N. Yusop, "Pareto ANOVA analysis for CMOS $0.18 \mu \mathrm{m}$ two-stage," Materials Science in Semiconductor Processing, vol. 24. pp. 9-14. 2014. [Online]. Available: https://www.sciencedirect.com/science/article/abs/pii/S1369800114001061.

[7] A. O. Ageev, A. E. Belyaev, N. S. Boltovets, V. N. Ivanov, R. V. Konakova, Ya. Ya. Kudrik, P. M. Litvin, V. V. Milenin, A. V. Sachenko, "Au-TiBx-n-6H-SiC Schottky barrier diodes: the features of current flow in rectifying and nonrectifying contacts," Semiconductors, vol. 43, no. 7. pp. 897-903, 2009. [Online]. Available: https://www.researchgate.net/publication/284573836_Au-TiBx-n-6H SiC_Schottky_barrier_diodes_The_features_of_current_flow_in_rectifying_and_nonrectifying_contacts.

[8] Z. Li, J. Waldron, T. Detchprohm, C. Wetzel, R. F. Karlicek, Jr. T. P. Chow, "Monolithic integration of lightemitting diodes and power metal-oxide- semiconductor channel high-electron-mobility transistors for light-emitting power integrated circuits in GaN on sapphire substrate," Applied Physics Letters, vol. 102 no. 19, pp. 192107192109. 2013. [Online]. Available: https://sci-hub.se/https://doi.org/10.1063/1.4807125.

[9] Jung-Hui Tsai, Shao-Yen Chiu, Wen-Shiung Lour, Der-Feng Guo, "High-performance InGaP/GaAs pnp $\delta$-doped heterojunction bipolar transistor," Semiconductors, vol. 43, pp. 939-942, 2009. [Online]. Available: https://link.springer.com/article/10.1134/S1063782609070227.

[10] S. R. Panda, A. Sahu, S. Das, A. K. Panda, T. Sahu, "Doping-Dependent Nonlinear Electron Mobility in GaAs $\mid \mathrm{In}_{\mathrm{x}}$ Ga1-xAs Coupled Quantum-Well Pseudo-Morphic MODFET Structure,” Semiconductors, vol. 54, pp. 788-795. 2020. [Online]. Available: https://link.springer.com/article/10.1134/S1063782620070118.

[11] M. J. Kumar, T. V. Singh, "Quantum confinement effects in strained silicon mosfets," International Journal of Nanoscience, vol. $7, \quad$ no. 2-3, pp. 81-84. 2008. [Online]. Available: https://www.worldscientific.com/doi/abs/10.1142/S0219581X08005195.

[12] P. Sinsermsuksakul, K. Hartman, S. B. Kim, J. Heo, L. Sun, H. H. Park, R. Chakraborty, T. Buonassisi, R. G. Gordon, "Enhancing the efficiency of SnS solar cells via band-offset engineering with a zinc oxysulfide buffer layer," Applied Physics Letters, vol. 102, no. 5, pp. 053901-053905. 2013. [Online]. Available: https://aip.scitation.org/doi/abs/10.1063/1.4789855.

[13] J. G. Reynolds, C. L. Reynolds, Jr. A. Mohanta, J. F. Muth, J. E. Rowe, H. O. Everitt, D. E. Aspnes, "Shallow acceptor complexes in p-type ZnO," Applied Physics Letters, vol. 102, no. 15, pp. 152114-152118. 2013. [Online]. Available: https://aip.scitation.org/doi/abs/10.1063/1.4802753.

[14] K. K. Ong, K. L. Pey, P. S. Lee, A. T. S. Wee, X. C. Wang, Y. F. Chong, "Dopant distribution in the recrystallization transient at the maximum melt depth induced by laser annealing," Applied Physics Letters, vol. 89, no. 17, pp. 172111-172114. 2006. [Online]. Available: https://aip.scitation.org/doi/abs/10.1063/1.2364834.

[15] H. T. Wang, L. S. Tan, E. F. Chor, "Pulsed laser annealing of Be-implanted GaN," Journal of Applied Physics, vol. 98, no. 9, pp. 094901-094905. 2006. [Online]. Available: https://aip.scitation.org/doi/abs/10.1063/1.2120893.

[16] S. T. Shishiyanu, T. S. Shishiyanu, S. K. Railyan, "Shallow p-n-junctions in Si prepared by pulse photon annealing," Semiconductors, vol. 36, no. 5, pp. 611-617. 2002. [Online]. Available: 
https://www.researchgate.net/publication/225941106_Shallow_p-

n_junctions_formed_in_silicon_using_pulsed_photon_annealing.

[17] Vladimir V. Kocharovsky, "Radiophysics and Quantum Electronics," Springer, vol. 43, no. 3, pp. 836-843. 2003. [Online]. Available: https://www.springer.com/journal/11141.

[18] Yu. S. Magda, "Modern microcontrollers. Architecture, programming, development of device, Radio and communication, Moscow, 2012.

[19] E. L. Pankratov, E. A. Bulaeva, "Doping of materials during manufacture p-n-junctions and bipolar transistors, Analytical approaches to model technological approaches and ways of optimization of distributions of dopants," American Scientific Publishers, vol. 1, no. 1, pp. 58-82. 2013. [Online]. Available: https://www.ingentaconnect.com/contentone/asp/rits/2013/00000001/00000001/art00004.

[20] V. V. Kozlivskii, V. A. Kozlov, V. N. Lamosav, "Modification of semiconductors by proton beams. A review," $\begin{array}{lllll}\text { Semicobductors, } & \text { vol. } 34, \quad \text { no. } 123-140, & 2000 . & \text { [Online]. }\end{array}$ https://link.springer.com/article/10.1134/1.1187921.

[21] Z. Yu. Gotra. Technology of microelectronic devices, Radio and communication, Moscow, 1991.

[22] V. L. Vinetskiy, G. A. Kholodar', Radiative physics of semiconductors. Naukova Dumka, Kiev, 1979.

[23] P. M. Fahey, P. B. Griffin, J. D. Plummer, "Point defects and dopant diffusion in silicon," Reviews Of Modern $\begin{array}{llllll}\text { Physics, } & \text { vol. } & 61, & \text { pp. } & \text { 289-388. } & \text { Available: }\end{array}$ https://journals.aps.org/rmp/abstract/10.1103/RevModPhys.61.289.

[24] A. N. Tikhonov, A. A. Samarskii. The mathematical physics equations, Science, Moscow, 1972.

[25] H. S. Carslaw, J. C. Jaeger. Conduction of heat in solids, Oxford University Press, 1964.

[26] E. L. Pankratov, "Dopant diffusion dynamics and optimal diffusion time as influenced by diffusion-coefficient nonuniformity," Russian Microelectronics, vol. 36, pp. 33-39, 2007. [Online]. Available: https://link.springer.com/article/10.1134/S1063739707010040.

[27] E. L. Pankratov, "On approach to optimize manufacturing of bipolar heterotransistors framework circuit of an operational amplifier to increase their integration rate. Influence mismatch-induced stress, Journal of Computational and Theoretical Nanoscience, vol. 14, no. 10, pp. 4885-4899. 2017. [Online]. Available: https://www.ingentaconnect.com/contentone/asp/jctn/2017/00000014/00000010/art00022.

[28] E. L. Pankratov, "On optimization of manufacturing of two-phase logic circuit based on heterostructures to increase density of their elements. Influence of miss-match induced stress," Advanced science, engineering and medicine, vol. $9, \quad$ no. $9, \quad$ pp. 787-801. 2017. [Online]. Available: https://www.ingentaconnect.com/contentone/asp/asem/2017/00000009/00000009/art00010.

[29] E. L. Pankratov, E. A. Bulaeva, "On increasing of density of transistors in a hybrid cascaded multilevel inverter," Multidiscipline Modeling in Materials and Structures, vol. 13, no. 4, pp. 664-677, 2017. [Online]. Available: https://www.emerald.com/insight/content/doi/10.1108/MMMS-05-2017-0041/full/html.

[30] E. L. Pankratov, E. A. Bulaeva, "An approach to manufacture of bipolar transistors in thin film structures. On the method of optimization," International Journal of Micro-Nano Scale Transport, vol. 4, no. 1, pp. 17-31. 2014. [Online]. Available: http://triggered.stanford.clockss.org/ServeContent?url=http://multiscience.atypon.com\%2Fdoi\%2Fpdf\%2F10.1260\%2F1759-3093.4.1-2.17.

[31] E. L. Pankratov, E. A. Bulaeva, "On increasing of density of transistors in a hybrid cascaded multilevel inverter," Multidiscipline Modeling in Materials and Structures, vol. 13, no. 4, pp. 578-604. 2017. [Online]. Available: https://www.emerald.com/insight/content/doi/10.1108/MMMS-05-2017-0041/full/html.

[32] E. L. Pankratov, E. A. Bulaeva, "An approach to increase the integration rate of planar drift heterobipolar transistors," Materials science in semiconductor processing, vol. 34, pp. 260-268. 2015. [Online]. Available: https://www.sciencedirect.com/science/article/abs/pii/S1369800115001365. 